\title{
Efficient estimation of multipartite quantum coherence
}

\author{
Qi-Ming Ding $\odot,{ }^{1, *}$ Xiao-Xu Fang $\odot,{ }^{1, *}$ Xiao Yuan, ${ }^{2}$ Ting Zhang, ${ }^{1}$ and He Lu $\oplus^{1, \dagger}$ \\ ${ }^{1}$ School of Physics, Shandong University, Jinan 250100, China \\ ${ }^{2}$ Stanford Institute for Theoretical Physics, Stanford University, Stanford, California 94305, USA
}

(Received 15 October 2020; revised 6 February 2021; accepted 17 May 2021; published 22 June 2021)

\begin{abstract}
Quantification of coherence lies at the heart of quantum information processing and fundamental physics. Exact evaluation of coherence measures generally needs a full reconstruction of the density matrix, which becomes intractable for large-scale multipartite systems. Here, we propose a systematic theoretical approach to efficiently estimating lower and upper bounds of coherence in multipartite states. Under the stabilizer formalism, the lower bound is determined by the spectrum estimation method with a small number of measurements, and the upper bound is determined by a single measurement. We verify our theory with a four-qubit optical quantum system. We experimentally implement various multiqubit entangled states, including the Greenberger-HorneZeilinger state, the cluster state, and the W state, and show how their coherences are efficiently inferred from measuring a few observables.
\end{abstract}

DOI: 10.1103/PhysRevResearch.3.023228

\section{INTRODUCTION}

Quantum coherence, being one of the defining features of quantum mechanics, underlies the fundamental phenomena of quantum interference and plays a significant role in physics and quantum information processing (QIP), such as quantum cryptography [1-3], quantum metrology [4,5], nanoscale thermodynamics [6-8], and energy transport in a biological system [9]. Based on the general framework of quantum resource theories [10-12], a systematic framework of coherence has been introduced $[13,14]$, based on which various coherence measures have been defined [14-26]. Meanwhile, the framework of coherence has been extended from a single party to the multipartite scenario with several applications, such as quantum state merging [27], coherence localization [28], and incoherence teleportation [29]. Studies of the interconversion between coherence and other multipartite nonclassical correlations, such as entanglement [15,30,31], discord [32], and nonlocality [33], also highlight the fundamental role of quantum coherence. With the rapid development of quantum hardware in realizing large-scale multipartite systems, the ability to efficiently quantify the coherence would thus offer an operationally meaningful benchmarking tool and benefit our understanding of QIP tasks.

Several experiments have been reported regarding the efficient detection of robustness of coherence [34,35]. However, the experimental detection of a general coherence measure, such as the relative entropy of coherence [14], is still missing. Theoretical proposals to estimate general multipartite

\footnotetext{
*These authors contributed equally to this work.

†luhe@sdu.edu.cn

Published by the American Physical Society under the terms of the Creative Commons Attribution 4.0 International license. Further distribution of this work must maintain attribution to the author(s) and the published article's title, journal citation, and DOI.
}

coherence without costly state tomography have also been proposed [36-38]. While the initial proposals need either copies of the prepared multipartite state [36] or complicated postprocessing [37], the spectrum estimation method was recently proposed [38], which only requires local measurements and easy-to-compute postprocessing. Nevertheless, the performance of the spectrum estimation method highly depends on the choice of the measurements, and how it works for a general multipartite state still needs further study. Moreover, existing works generally focus on the lower bound of coherence. For a given quantum state, the maximal entanglement or discord it can generate is upper bounded by its coherence $[15,32]$, which makes detecting the upper bound of coherence important as it can indicate whether the given quantum state can generate sufficient resources for a certain QIP task.

In this paper, we theoretically address these issues by proposing two methods that can respectively detect the lower and upper bound of coherence for all multiqubit stabilizer states. The lower bound detection is based on the spectrum estimation method [38] and the stabilizer theory [39,40], which only requires a few local observable measurements for stabilizer states. The upper bound detection is based on the monogamy of coherence with a single local measurement. Experimentally, we prepare five stabilizer states of up to four qubits and demonstrate how a small number of measurements could enable us to infer multipartite coherence.

\section{THEORY}

\section{A. Lower bound estimation}

Under the computational basis $\left\{|i\rangle: i \in\{0,1\}^{\otimes n}\right\}$ of an $n$-qubit state, we consider the relative entropy of coherence [14]

$$
C_{\mathrm{RE}}(\rho)=S_{\mathrm{VN}}\left(\rho_{d}\right)-S_{\mathrm{VN}}(\rho),
$$

with $S_{\mathrm{VN}}=-\operatorname{tr}\left[\rho \log _{2} \rho\right]$ being the von Neumann entropy and $\rho_{d}=\sum_{i}\langle i|\rho| i\rangle|i\rangle\langle i|$ being the diagonal part of $\rho$. The 
relative entropy of coherence characterizes the asymptotic distillable coherence under different types of incoherent operations [41,42], quantifies the genuine randomness that can be extracted from measuring the quantum state in the computational basis [43-45], captures the deviation from thermodynamic equilibrium [46], etc. We thus focus on the estimation, in particular, the lower and upper bounds, of the relative entropy of coherence for general multipartite states.

The lower bound $l^{c}(\rho)$ of the coherence $C_{\mathrm{RE}}(\rho)$ can be obtained by spectrum estimation and the majorization theory [47] as

$$
C_{\mathrm{RE}}(\rho) \geqslant l^{c}(\rho)=S_{\mathrm{VN}}(\boldsymbol{d})-S_{\mathrm{VN}}\left[\boldsymbol{d} \vee\left(\wedge_{\boldsymbol{p} \in X} \boldsymbol{p}\right)\right],
$$

where $\boldsymbol{d}=\left(d_{1}, \ldots, d_{2^{n}}\right)$ are the diagonal elements of $\rho, \boldsymbol{p}=$ $\left(p_{1}, \ldots, p_{2^{n}}\right)$ is the estimated probability distribution of the measurement on a certain entangled basis $\left\{\left|\psi_{k}\right\rangle\right\}_{k=1}^{2^{n}}, \vee$ is the majorization join, and $\wedge_{\boldsymbol{p} \in X} \boldsymbol{p}$ is the majorization meet of all probability distributions in $X$ [38]. Here, the majorization join and meet are defined based on majorization. Specifically, given two probability distributions $\boldsymbol{a}=\left(a_{1}, a_{2}, \ldots, a_{n}\right)$ and $\boldsymbol{b}=\left(b_{1}, b_{2}, \ldots, b_{n}\right)$ with $a_{1} \geqslant a_{2} \geqslant \cdots \geqslant a_{n}$ and $b_{1} \geqslant b_{2} \geqslant$ $\ldots \geqslant b_{n}, \boldsymbol{a}$ is majorized by $\boldsymbol{b}$ (written as $\boldsymbol{a} \prec \boldsymbol{b}$ ) if it satisfies $\sum_{i=1}^{k} a_{i} \leqslant \sum_{i=1}^{k} b_{i}$ for all $k=1,2, \ldots, n$. A probability distribution $\boldsymbol{c}$ is called the majorization join (meet) of $\boldsymbol{a}$ and $\boldsymbol{b}$ if it satisfies (i) $c \succ \boldsymbol{a}, \boldsymbol{b}(\boldsymbol{c} \prec \boldsymbol{a}, \boldsymbol{b})$ and (ii) $c \prec \tilde{\boldsymbol{c}}(\boldsymbol{c} \succ \tilde{\boldsymbol{c}})$ for any $\tilde{\boldsymbol{c}}$ that satisfies $\boldsymbol{a}, \boldsymbol{b} \prec \tilde{\boldsymbol{c}}(\boldsymbol{a}, \boldsymbol{b} \succ \tilde{\boldsymbol{c}})$ [47]. Here, we consider that $\boldsymbol{p}$ is selected from the set $X$, which satisfies $X=\{\boldsymbol{p} \mid A \boldsymbol{p} \geqslant$ $\boldsymbol{\alpha}, B \boldsymbol{p}=\boldsymbol{\beta}\}$. $A$ and $B$ are matrices and $\boldsymbol{\alpha}$ and $\boldsymbol{\beta}$ are vectors. " $\geqslant "$ represents componentwise comparison.

To calculate $l^{c}(\rho)$ via Eq. (2), it is crucial to set constraints $A \boldsymbol{p} \geqslant \boldsymbol{\alpha}$ and $B \boldsymbol{p}=\boldsymbol{\beta}$ with experimentally collected data and then find the "largest" distribution $\boldsymbol{p}$ majorized by all probability distributions in $X$, i.e., $\wedge_{\boldsymbol{p} \in X} \boldsymbol{p}$. According to the Hermiticity of density matrix $\rho$, we can set $A$ as a $2^{n}$ dimensional identity matrix and $\alpha=0$ for $A \boldsymbol{p} \geqslant \boldsymbol{\alpha}$, by which $p_{k} \geqslant 0$ is guaranteed. In the following section, we introduce the procedure to construct constraint $B \boldsymbol{p}=\boldsymbol{\beta}$ via stabilizer formalism.

\section{B. Constructing constraint via stabilizer formalism}

An observable $S_{i}$ stabilizes an $n$-qubit state $|\psi\rangle$ if $|\psi\rangle$ is an eigenstate with eigenvalue +1 of $S_{i}$, i.e., $S_{i}|\psi\rangle=|\psi\rangle$. The set $\mathcal{S}$ of operators $S_{i}$ is the stabilizer of $|\psi\rangle$, and $|\psi\rangle$ is a socalled stabilizer state $[40,48]$. For an $n$-qubit state, there are $n$ stabilizing operators $\left\{S_{1}, \ldots, S_{n}\right\}$ that can uniquely determine $|\psi\rangle$. Here, $S_{1}, \ldots, S_{n}$ are the generators of the set $\mathcal{S}$, and we denote $\mathcal{S}=\left\langle S_{1}, \ldots, S_{n}\right\rangle$. Note that $|\psi\rangle$ is stabilized not only by $\left\{S_{1}, \ldots, S_{n}\right\}$, but also by their products. Thus there could be in total $2^{n}$ stabilizer operators in $\mathcal{S}$.

Given an $n$-qubit stabilizer state $\left|\psi_{1}\right\rangle$ associated with stabilizer $\mathcal{S}$, there exists an orthonormal basis $\left\{\left|\psi_{k}\right\rangle\right\}_{k=1}^{2^{n}}$ including $\left|\psi_{1}\right\rangle=|\psi\rangle$, where $\left|\psi_{k}\right\rangle$ is uniquely specified by $\mathcal{S}$ but with different eigenvalues, i.e., $S_{i}\left|\psi_{k}\right\rangle=a_{i k}\left|\psi_{k}\right\rangle$ with eigenvalues $a_{i k}= \pm 1$. For example, the Bell state $\left|\Phi^{+}\right\rangle=(|00\rangle+$ $|11\rangle) / \sqrt{2}$ can be specified by $\mathcal{S}=\left\langle X^{(1)} X^{(2)}, Z^{(1)} Z^{(2)}\right\rangle$. Hereinafter, $X^{(j)}, Y^{(j)}$, and $Z^{(j)}$ denote the Pauli matrices $\sigma_{x}, \sigma_{y}$, and $\sigma_{z}$ acting on the $j$ th qubit. In the following, qubit index $j$ may be omitted if there is no confusion. The four Bell states $\left|\Phi^{ \pm}\right\rangle$and $\left|\Psi^{ \pm}\right\rangle$are specified by $\mathcal{S}=\langle X X, Z Z\rangle$ as well, with eigenvalues of $(+1,+1),(-1,+1),(+1,-1)$, and $(-1,-1)$, respectively. The basis $\left\{\left|\psi_{k}\right\rangle\right\}_{k=1}^{2^{n}}$ associated with the same stabilizer $\mathcal{S}$ is also called the graph-diagonal basis [49,50] as a stabilizer state is equivalent to a graph state under local Clifford operations [51]. In the graph-diagonal basis, the stabilizing operator can be written as $S_{i}=\sum_{k} a_{i k}\left|\psi_{k}\right\rangle\left\langle\psi_{k}\right|$, and its expected value on a given quantum state $\rho$ is $\left\langle S_{i}\right\rangle=$ $\operatorname{tr}\left(S_{i} \rho\right)=\sum_{k} p_{k} a_{i k}$, where the parameters $p_{k}=\left\langle\psi_{k}|\rho| \psi_{k}\right\rangle$ form a probability distribution $\boldsymbol{p}=\left(p_{1}, \ldots, p_{2^{n}}\right)$ with $p_{k} \geqslant 0$ and $\sum_{k} p_{k}=1$.

The expected values of stabilizer $\mathcal{S}$ on $\rho$ lead to $2^{n}$ equations and can be represented in matrix form as

$$
\underbrace{\left[\begin{array}{ccc}
a_{11} & \ldots & a_{12^{n}} \\
\vdots & \ddots & \vdots \\
a_{2^{n} 1} & \cdots & a_{2^{n} 2^{n}}
\end{array}\right]}_{B} \cdot \underbrace{\left[\begin{array}{c}
p_{1} \\
\vdots \\
p_{2^{n}}
\end{array}\right]}_{\boldsymbol{p}}=\underbrace{\left[\begin{array}{c}
\left\langle S_{1}\right\rangle \\
\vdots \\
\left\langle S_{2^{n}}\right\rangle
\end{array}\right]}_{\boldsymbol{\beta}},
$$

from which we can construct the constraint $B \boldsymbol{p}=\boldsymbol{\beta}$. However, in practice, there do not always exist solutions of $\wedge_{\boldsymbol{p} \in X} \boldsymbol{p}$ in Eq. (2) with constraint equation (3) (as reflected by our experimental results). The experimentally generated state always has a distance to the target state due to the inevitable imperfections, which might lead to no solution of $\wedge_{\boldsymbol{p} \in X} \boldsymbol{p}$ with inputs of $\left\{\left\langle S_{i}\right\rangle\right\}$. On the other hand, experimentally obtained $\left\langle S_{i}\right\rangle$ is always associated with statistical errors. We address these issues by introducing the experimental standard deviation $\sigma_{i}$ of $\left\langle S_{i}\right\rangle$ and relax the constraint equation (3) to an inequality form,

$$
\underbrace{\left[\begin{array}{c}
\left\langle S_{1}\right\rangle-w \sigma_{1} \\
\vdots \\
\left\langle S_{2^{n}}\right\rangle-w \sigma_{2^{n}}
\end{array}\right]}_{\boldsymbol{\beta}_{-}} \leqslant B \cdot \boldsymbol{p} \leqslant \underbrace{\left[\begin{array}{c}
\left\langle S_{1}\right\rangle+w \sigma_{1} \\
\vdots \\
\left\langle S_{2^{n}}\right\rangle+w \sigma_{2^{n}}
\end{array}\right]}_{\boldsymbol{\beta}_{+}},
$$

where $w \sigma_{i}$ with $w \geqslant 0$ is the deviation from the mean value $\left\langle S_{i}\right\rangle$ represented in $\sigma_{i}$. To this end, an experimentally accessible constraint is formulated as $\boldsymbol{\beta}_{-} \leqslant B \boldsymbol{p} \leqslant \boldsymbol{\beta}_{+}$. In practice, instead of measuring all the stabilizers, which is impractical for a large quantum state, we can select a small subset of stabilizers so that the number of measurements does not scale exponentially with the number of qubits. Note that $\left\langle\mathbb{I}^{n}\right\rangle=1$ must be set in Eq. (4) to ensure $\sum_{k} p_{k}=1$. If we apply the scheme on the graph-diagonal states $\rho=\sum_{k} \lambda_{k}\left|\psi_{k}\right\rangle\left\langle\psi_{k}\right|$, with $\lambda=\left(\lambda_{1}, \ldots, \lambda_{2^{n}}\right)$ being the spectrum of $\rho$, we have $\boldsymbol{p}=\lambda$. Thus $\boldsymbol{d} \vee\left(\wedge_{\boldsymbol{p} \in X} \boldsymbol{p}\right)=\lambda$ implies $l^{c}(\rho)=C_{\mathrm{RE}}(\rho)$, which indicates that the estimated lower bound of coherence is tight for graph-diagonal states.

We emphasize that relaxing the constraint to $\boldsymbol{\beta}_{-} \leqslant B \boldsymbol{p} \leqslant$ $\boldsymbol{\beta}_{+}$does not increasing the risk of overestimation of $l^{c}(\rho)$. Suppose that $X_{1}$ and $X_{2}$ are two feasible sets of probability distributions and satisfy $X_{1} \subseteq X_{2} . X_{1}$ and $X_{2}$ are restricted to $\boldsymbol{d} \prec \wedge_{\boldsymbol{p} \in X_{1}} \boldsymbol{p}$ and $\boldsymbol{d} \prec \wedge_{\boldsymbol{p} \in X_{2}} \boldsymbol{p}$; otherwise the result of Eq. (2) is 0 . According to the definition of the majorization meet, $\wedge_{\boldsymbol{p} \in X} \boldsymbol{p}$ is the "largest" distribution majorized by all probability distributions in $X$. Therefore $\wedge_{\boldsymbol{p} \in X} \boldsymbol{p}$ becomes "smaller" when we enlarge the range of $X$, i.e., $\wedge_{\boldsymbol{p} \in X_{1}} \boldsymbol{p} \succ \wedge_{\boldsymbol{p} \in X_{2}} \boldsymbol{p}$ for $X_{1} \subseteq$ $X_{2}$. According to the strict Schur concavity of the Shannon entropy $S$, we obtain $S\left(\wedge_{\boldsymbol{p} \in X_{1}} \boldsymbol{p}\right)<S\left(\wedge_{\boldsymbol{p} \in X_{2}} \boldsymbol{p}\right)$, which implies 
$S(\boldsymbol{d})-S\left(\wedge_{\boldsymbol{p} \in X_{1}} \boldsymbol{p}\right)>S(\boldsymbol{d})-S\left(\wedge_{\boldsymbol{p} \in X_{2}} \boldsymbol{p}\right)$. Thus we conclude that $l^{c}(\rho)$ decreases with enlarging range of $X$.

Similar constraints can also be formulated for multiqubit states that do not obviously fit the stabilizer formalism. The stabilizing operators of such kind of $n$-qubit states $|\psi\rangle$ could be determined by finding its unitary dynamics $U^{\psi}$ acting on $|0\rangle^{\otimes n}$, i.e., $|\psi\rangle=U^{\psi}|0\rangle^{\otimes n}$ [48]. As $|0\rangle^{\otimes n}$ is stabilized by $S_{i}^{|0\rangle^{\otimes n}}=Z^{(i)}, \forall i \in\{1,2, \ldots, n\}$, the stabilizing operator of $|\psi\rangle$ is $S_{i}^{\psi}=U^{\psi} Z^{(i)} U^{\psi^{\dagger}}$.

\section{Upper bound estimation}

Theorem 1. Let $\mathcal{M}(\boldsymbol{d})$ be a set of states with the same diagonal part $\boldsymbol{d}=\left(d_{1}, \ldots, d_{2^{n}}\right)$; then $\left|\psi_{d}\right\rangle=\sum_{i=1}^{2^{n}} \sqrt{d_{i}}|i\rangle$ is the maximally coherent state in $\mathcal{M}(\boldsymbol{d})$.

Proof. It is equivalent to prove that $\left|\psi_{d}\right\rangle=\sum_{i=1}^{2^{n}} \sqrt{d_{i}}|i\rangle$ can be transformed into any $\rho \in \mathcal{M}(\boldsymbol{d})$ via incoherent operation.

We first consider the case in which there are no nonzero elements in $\boldsymbol{d}$, i.e., $d_{i} \neq 0$ for all $i$. Let $\left\{\lambda_{\alpha},\left|\varphi_{\alpha}\right\rangle\right\}$ be the spectral decomposition of $\rho$, i.e.,

$$
\rho=\sum_{\alpha} \lambda_{\alpha}\left|\varphi_{\alpha}\right\rangle\left\langle\varphi_{\alpha}\left|=\sum_{\alpha} \lambda_{\alpha} \sum_{i, j} c_{\alpha i} c_{\alpha j}^{*}\right| i\right\rangle\langle j|,
$$

where $\left|\varphi_{\alpha}\right\rangle=\sum_{i=1}^{2^{n}} c_{\alpha i}|i\rangle$ with $\sum_{i=1}^{2^{n}}\left|c_{\alpha i}\right|^{2}=1$ for all $\alpha$. Thus $\rho$ form a set $\mathcal{M}(\boldsymbol{d})$ with $d_{i}=\sum_{\alpha} \lambda_{\alpha}\left|c_{\alpha i}\right|^{2}$. We define a completely positive and trace-preserving (CPTP) map $\Lambda_{1}(\cdot)=\sum_{\alpha} K_{\alpha} \cdot K_{\alpha}^{\dagger}$ associated with Kraus operators $K_{\alpha}=$ $\sum_{i} \frac{\sqrt{\lambda_{\alpha}} c_{\alpha i}}{\sqrt{d_{i}}}|i\rangle\langle i|$, which transforms $\left|\psi_{d}\right\rangle\left\langle\psi_{d}\right|$ into $\rho$ by

$$
\begin{aligned}
\Lambda_{1}\left(\left|\psi_{d}\right\rangle\left\langle\psi_{d}\right|\right) & =\sum_{\alpha} K_{\alpha}\left|\psi_{d}\right\rangle\left\langle\psi_{d}\right| K_{\alpha}^{\dagger} \\
& =\sum_{\alpha} \sum_{i, j, k, l} \frac{\lambda_{\alpha} c_{\alpha i} c_{\alpha j}^{*}}{\sqrt{d_{i} d_{j}}} \sqrt{d_{k} d_{l}} \delta_{i k} \delta_{j l}|i\rangle\langle j| \\
& =\sum_{\alpha} \lambda_{\alpha} \sum_{i, j} c_{\alpha i} c_{\alpha j}^{*}|i\rangle\langle j| \\
& =\rho .
\end{aligned}
$$

According to the definition of strictly incoherent operation $[52,53], K_{\alpha}$ is a strictly incoherent operator, and $\Lambda_{1}(\cdot)$ is a strictly incoherent operation.

For the case in which there are $m\left(m<2^{n}\right)$ nonzero elements in $\boldsymbol{d}$, we first transform $\left|\psi_{d}\right\rangle\left\langle\psi_{d}\right|$ into a block-diagonal matrix via a permutation matrix $M$; that is, $M\left|\psi_{d}\right\rangle\left\langle\psi_{d}\right| M^{-1}=$ $\left|\psi_{m}\right\rangle\left\langle\psi_{m}\left|\oplus \mathbf{0}_{2^{n}-m}.\right| \psi_{m}\right\rangle=\sum_{i^{\prime}=1}^{m} \sqrt{d_{i^{\prime}}}\left|i^{\prime}\right\rangle$ is the vector in $m-$ dimensional Hilbert space spanned by $\left\{\left|i^{\prime}\right\rangle\right\}$, where $i^{\prime}$ is the rearranged index of $m$ nonzero elements. $\mathbf{0}$ is a $\left(2^{n}-m\right) \times$ $\left(2^{n}-m\right)$ all-0 matrix.

Similarly, we define the CPTP map $\Lambda_{2}(\cdot)=\sum_{\beta} K_{\beta} \cdot K_{\beta}^{\dagger}$ associated with Kraus operators $K_{\beta}=\sum_{i^{\prime}} \frac{\sqrt{\lambda_{\beta}} c_{\beta i^{\prime}}}{\sqrt{d_{i^{\prime}}}}\left|i^{\prime}\right\rangle\left\langle i^{\prime}\right|$ acting on $m$-dimensional Hilbert space. Thus we can derive that

$$
M^{-1} \Lambda_{2}\left(M\left|\psi_{d}\right\rangle\left\langle\psi_{d}\right| M^{-1}\right) M=\rho .
$$

Note that any permutation matrix is strictly incoherent unitary [54]. For any two strictly incoherent operations $\Lambda_{\alpha}$ and $\Lambda_{\beta}$, the operation $\Lambda=\Lambda_{\alpha} \circ \Lambda_{\beta}$ is also a strictly incoherent operation [55]. Thus $\left|\psi_{d}\right\rangle=\sum_{i=1}^{2^{n}} \sqrt{d_{i}}|i\rangle$ can be transformed into any $\rho \in \mathcal{M}(\boldsymbol{d})$ via strictly incoherent operations $M^{-1} K_{\beta} M$.

To find the upper bound of the coherence of an $n$-qubit state $\rho$, we measure it in the computational basis $\{|i\rangle\}$, which yields a distribution $\boldsymbol{d}=\left(d_{1}, \ldots, d_{2^{n}}\right)$. Based on the monogamy of the relative entropy of coherence [14], the coherence of $\rho$ is upper bounded by the coherence $u_{c}(\rho)$ of state $\left|\psi_{d}\right\rangle=$ $\sum_{i=1}^{2^{n}} \sqrt{d_{i}}|i\rangle$, i.e.,

$$
C_{\mathrm{RE}}(\rho) \leqslant u_{c}(\rho)=C_{\mathrm{RE}}\left(\left|\psi_{d}\right\rangle\left\langle\psi_{d}\right|\right) .
$$

Note that this upper bound is tight for pure states and can be used for various coherence measures.

\section{EXPERIMENTAL DEMONSTRATION}

Next, we demonstrate the capability of our scheme by estimating the coherence of several typical multiqubit states. We firstly generate photon pairs by a periodically poled potassium
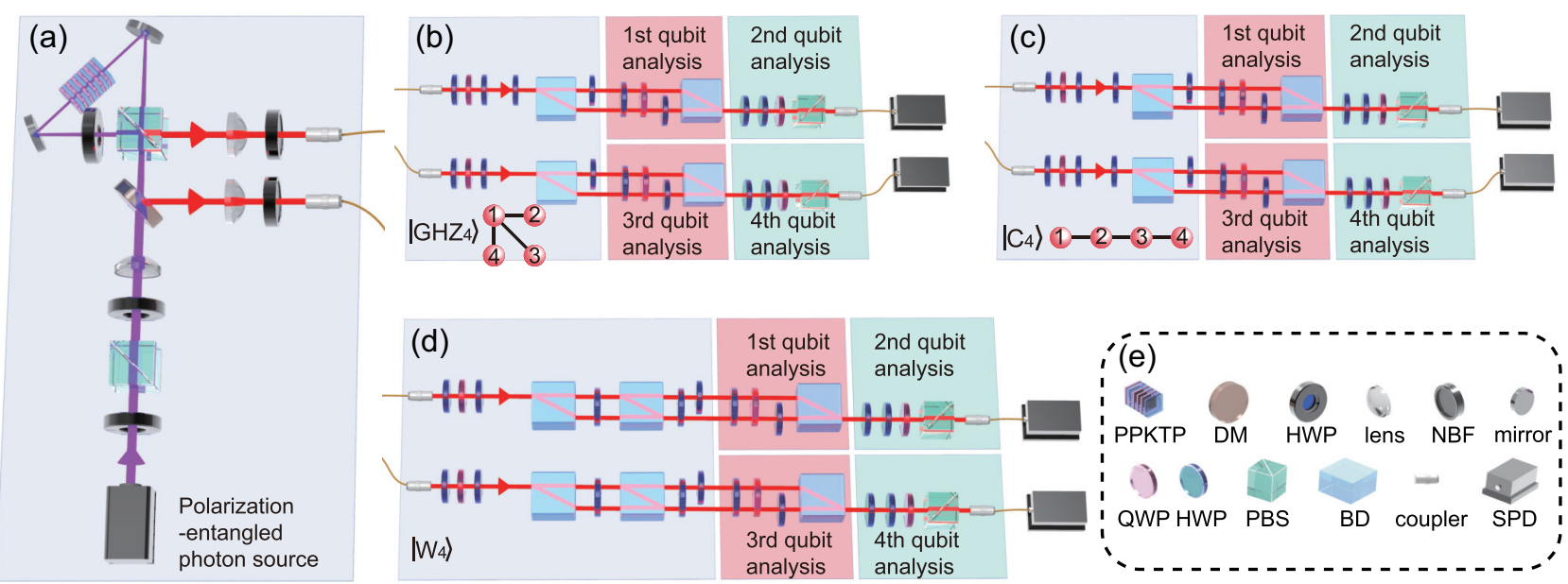

FIG. 1. Schematic drawing of the experimental setup. (a) The setup to generate a polarization-entangled photon pair. (b)-(d) The setups to generate $\left|\mathrm{GHZ}_{4}\right\rangle,\left|\mathrm{C}_{4}\right\rangle$, and $\left|\mathrm{W}_{4}\right\rangle$, respectively. (e) Symbols used in (a)-(d): PPKTP, periodically poled potassium titanyl phosphate; DM, dichroic mirror; HWP, half-wave plate; NBF, narrowband filter; QWP, quarter-wave plate; PBS, polarization beam splitter; BD, beam displacer; SPD, single-photon detector. More experimental details can be found in Appendix B. 
titanyl phosphate (PPKTP) crystal in a Sagnac interferometer [56], which is bidirectionally pumped by an ultraviolet (UV) laser diode with central wavelength at $405 \mathrm{~nm}$ [as shown in Fig. 1(a)]. The two photons are entangled in the polarization degree of freedom (DOF), i.e., $\left|\Psi^{+}\right\rangle=(|H V\rangle+|V H\rangle) / \sqrt{2}$ with $H$ being the horizontal polarization and $V$ being the vertical polarization. We extend the photon to its path DOF using a beam displacer (BD), which transmits vertical polarization and deviates horizontal polarization, i.e., $|H\rangle \rightarrow|H\rangle|h\rangle$ and $|V\rangle \rightarrow|V\rangle|v\rangle$ with $h$ and $v$ being the path DOF [57-59]. The qubit is encoded in the polarization DOF as $|H(V)\rangle \rightarrow$ $|0(1)\rangle$ and in the path DOF as $|h(v)\rangle \rightarrow|0(1)\rangle$. In our experiment, we denote the qubits encoded in the polarization DOF as 1 and 3, while the qubits encoded in the path DOF are denoted as 2 and 4. As shown in Figs. 1(b)-1(d), with different experimental setup configurations, we can generate various four-qubit states, including $\left|\mathrm{GHZ}_{4}\right\rangle=(|0000\rangle+$ $|1111\rangle) / \sqrt{2}$ [a Greenberger-Horne-Zeilinger (GHZ) state], $\left|\mathrm{C}_{4}\right\rangle=(|0000\rangle+|0011\rangle+|1100\rangle-|1111\rangle) / 2$, and $\left|\mathrm{W}_{4}\right\rangle=$ $(|0001\rangle+|0010\rangle+|0100\rangle+|1000\rangle) / 2$. Moreover, $|\mathrm{GHZ}\rangle_{3}=$ $(|000\rangle+|111\rangle) / \sqrt{2}$ and $\left|\mathrm{W}_{3}\right\rangle=(|100\rangle+|010\rangle+|001\rangle) /$ $\sqrt{3}$ can be obtained by extending one photon to polarization and path DOFs while keeping the other in the polarization DOF (see Appendix B for more details).

For each experimentally generated state $\rho_{\text {expt }}^{\psi}$, we measure the expected values of its stabilizers $\left\langle S_{i}\right\rangle$ associated with the corresponding statistical errors $\sigma_{i}$. We refer the reader to Appendix A for details of stabilizing operators of $|\psi\rangle$ and the corresponding graph-diagonal basis. The measured expected values of stabilizing operators are presented in Appendix B. With the measured $\left\langle S_{i}\right\rangle$ and $\sigma_{i}$, we construct the constraint Eq. (4). Thus we can calculate $l_{\omega, m}^{c}\left(\rho_{\text {expt }}^{\psi}\right)$ via solving Eq. (2), where $\omega$ represents the setting of deviations and $m$ is the number of $\left\langle S_{i}\right\rangle$ with which we construct the constraint. We set $\omega$ as non-negative integers from 0 to 3 , and $m$ from 1 to $2^{n}-1$ as $\left\langle\mathbb{I}^{n}\right\rangle=1$ must be set in the constraint. In our calculation, we treat the case of no solution as $l_{\omega, m}^{c}\left(\rho_{\text {expt }}^{\psi}\right)=0$ and the case of $l_{\omega, m}^{c}\left(\rho_{\text {expt }}^{\psi}\right)>0$ as a valid solution. For a fixed $m$, there are $\left(2^{2^{n}}-1\right)$ subsets of $\left\{S_{i}^{\psi}\right\}$, and the maximum $l_{\omega, m}^{c}\left(\rho_{\text {expt }}^{\psi}\right)$ is shown in Fig. 2. We observe that valid $l_{\omega, m}^{c}\left(\rho_{\text {expt }}^{\psi}\right)$ cannot be obtained with $m \leqslant 3$ stabilizers for $\rho_{\text {expt }}^{\mathrm{GHZ}_{4}, m \leqslant 1}$ stabilizers for $\rho_{\text {expt }}^{\mathrm{W}_{3}}$, and $m \leqslant 2$ stabilizers for the other three states. With increase in $m$, the maximum $l_{\omega, m}^{c}\left(\rho_{\text {expt }}^{\psi}\right)$ increases accordingly. When $m$ gets close to $2^{n}-1$, there exist situations in which we cannot obtain $l_{\omega, m}^{c}\left(\rho_{\text {expt }}^{\psi}\right)$ for smaller $w$ [the values are 0 in the lower right corner of Figs. 2(b)-2(e)]. As previously mentioned, this is caused by experimental imperfections, such as slight misalignment of optical elements during data collection, which introduces small variation in prepared $\rho_{\text {expt }}^{\psi}$. The issue is improved by extending the range of $\left\langle S_{i}^{\psi}\right\rangle$, i.e., increasing $\omega$. As shown in Figs. 2(b)-2(e), most $l_{\omega, m}^{c}\left(\rho_{\text {expt }}^{\psi}\right)$ have valid solutions for large $m$ by setting $\omega=$ 3. Moreover, the accuracy of estimated $l_{\omega, m}^{c}\left(\rho_{\mathrm{expt}}^{\psi}\right)$ increases along with $m$ as well. We investigate this by calculating the normalized distance between $l_{\omega, m}^{c}\left(\rho_{\text {expt }}^{\psi}\right)$ and $C_{\mathrm{RE}}\left(\rho_{\text {expt }}^{\psi}\right)$, i.e., $1-l_{\omega, m}^{c}\left(\rho_{\text {expt }}^{\psi}\right) / C_{\mathrm{RE}}\left(\rho_{\text {expt }}^{\psi}\right)$. $C_{\mathrm{RE}}\left(\rho_{\text {expt }}^{\psi}\right)$ is calculated with
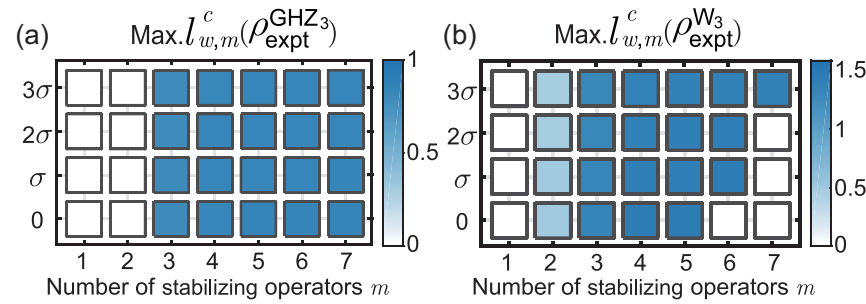

(c)

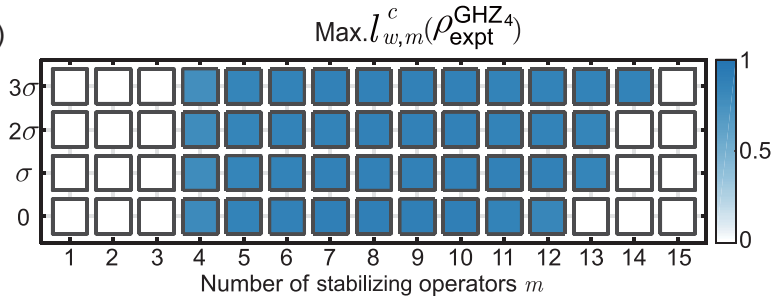

(d)

$$
\operatorname{Max} . l_{w, m}^{c}\left(\rho_{\text {expt }}^{\mathrm{C}_{4}}\right)
$$

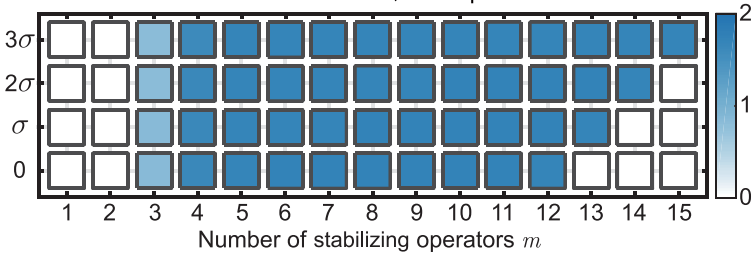

(e)

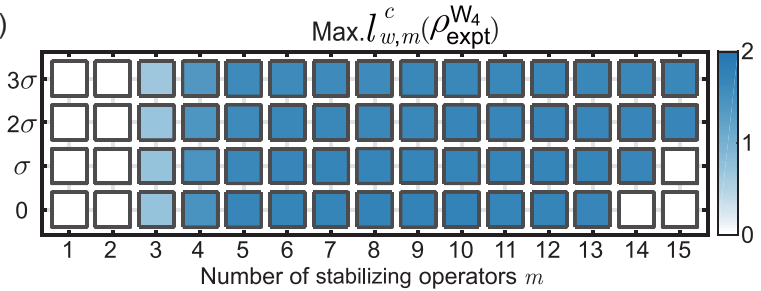

FIG. 2. (a)-(e) Experimental results of $l_{\omega, m}^{c}\left(\rho_{\mathrm{expt}}^{\psi}\right)$. The color bar indicates the range of $l_{\omega, m}^{c}\left(\rho_{\text {expt }}^{\psi}\right)$ from 0 to values of ideal $|\psi\rangle$, i.e., 1 for $\left|\mathrm{GHZ}_{3}\right\rangle$ and $\left|\mathrm{GHZ}_{4}\right\rangle, 1.585$ for $\left|\mathrm{W}_{3}\right\rangle$, and 2 for $\left|\mathrm{C}_{4}\right\rangle$ and $\left|\mathrm{W}_{4}\right\rangle$.

Eq. (1) by reconstructing $\rho_{\text {expt }}^{\psi}$ via quantum state tomographic technology (see Appendix B for the reconstructed $\rho_{\text {expt }}^{\psi}$ ). The distances between maximal $l_{3, m}^{c}\left(\rho_{\text {expt }}^{\psi}\right)$ and $C_{\mathrm{RE}}\left(\rho_{\text {expt }}^{\psi}\right)$ are shown in Fig. 3(a), from which we observe that the distance drops down quickly with increasing $m$ and tends to converge at $m=5$.

As previously mentioned, the choice of selecting $m$ stabilizers from $\left\{S_{i}^{\psi}\right\}$ is not unique except $m=2^{n}-1$. An important property is the successful probability of obtaining valid $l_{\omega, m}^{c}$ by randomly selecting $m$ stabilizers. We show the percentage of valid $l_{\omega, 5}^{c}\left(\rho_{\text {expt }}^{\psi}\right)\left[l_{\omega, 5}^{c}\left(\rho_{\text {expt }}^{\psi}\right)>0\right]$ for $m=$ 5 in Fig. 3(b). By increasing $\omega$, the probability of getting valid $l_{\omega, 5}^{c}\left(\rho_{\text {expt }}^{\psi}\right)$ is enhanced, especially for $\rho_{\text {expt }}^{\mathrm{W}_{3}}$. However, one cannot increase $\omega$ arbitrarily. A larger $\omega$ represents a smaller probability that we could obtain $\left\langle S_{i}\right\rangle$ in the range $\left[\left\langle S_{i}\right\rangle+(w-1) \sigma_{i},\left\langle S_{i}\right\rangle+w \sigma_{i}\right]$ as well as $\left[\left\langle S_{i}\right\rangle-w \sigma_{i},\left\langle S_{i}\right\rangle-\right.$ $\left.(w-1) \sigma_{i}\right]$, which is less than $0.3 \%$ for $\omega=3$. This is also reflected by the normalized distance of maximal $l_{\omega, 5}^{c}\left(\rho_{\text {expt }}^{\psi}\right)$ shown in Fig. 3(c), which indicates that the inaccuracy of $l_{\omega, 5}^{c}\left(\rho_{\text {expt }}^{\psi}\right)$ increases when we extend the range of $\left\langle S_{i}\right\rangle$. Also, the results in Fig. 3(c) agree with our claim that relaxing the constraint decreases the estimated value of $l^{c}(\rho)$. We conclude that setting $\omega=3$ is reasonable in experiment, under which 

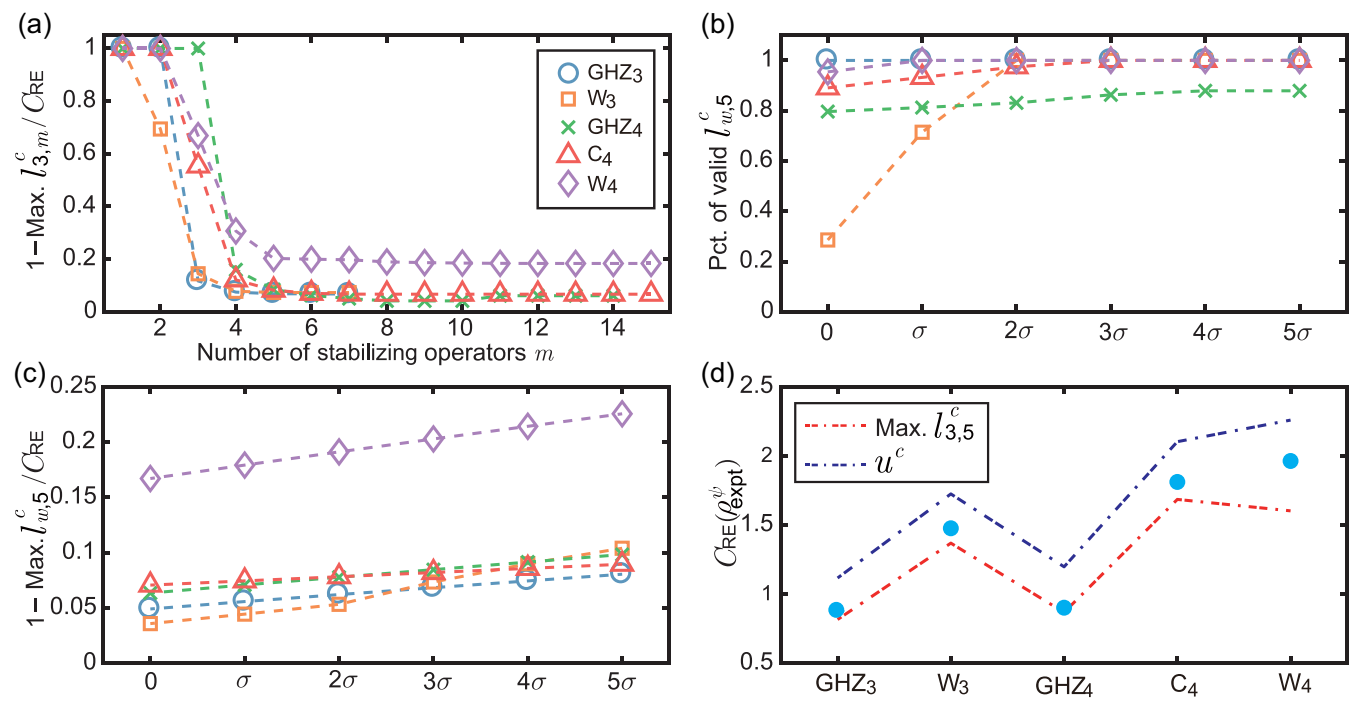

FIG. 3. (a) The normalized distance between maximal $l_{3, m}^{c}\left(\rho_{\text {expt }}^{\psi}\right)$ and $C_{\mathrm{RE}}\left(\rho_{\mathrm{expt}}^{\psi}\right)$. (b) The percentage (Pct.) of valid $l_{\omega, 5}^{c}$ obtained over all $\left(\begin{array}{c}2^{n}-1 \\ 5\end{array}\right)$ subsets. (c) The normalized distance between maximal $l_{\omega, 5}^{c}\left(\rho_{\mathrm{expt}}^{\psi}\right)$ and $C_{\mathrm{RE}}\left(\rho_{\mathrm{expt}}^{\psi}\right)$ of setting $\omega$ from 0 to 5 . (d) The $u^{c}$ (blue dashdotted line) for $\rho_{\text {expt }}^{\mathrm{GHZ}_{3}}, \rho_{\text {expt }}^{\mathrm{W}_{3}}, \rho_{\text {expt }}^{\mathrm{GHZ}_{4}}, \rho_{\text {expt }}^{\mathrm{C}_{4}}$, and $\rho_{\text {expt }}^{\mathrm{W}_{4}}$ are $1.117 \pm 0.003,1.725 \pm 0.004,1.198 \pm 0.005,2.103 \pm 0.004,1.725 \pm 0.004$, and $2.259 \pm 0.006$, respectively. The corresponding maximal $l_{\omega, m}^{c}$ (red dash-dotted line) are the results of $\omega=3$ and $m=5$. The solid circles represent $C_{\mathrm{RE}}\left(\rho_{\mathrm{expt}}^{\psi}\right)$, and their error bars are too small compared with the marker size [see Appendix B for the values of $C_{\mathrm{RE}}\left(\rho_{\mathrm{expt}}^{\psi}\right)$ ].

we observe that the probability of getting valid $l_{\omega, 5}^{c}\left(\rho_{\text {expt }}^{\psi}\right)$ is $100 \%$ for $\rho_{\text {expt }}^{\mathrm{GHZ}_{3}}, \rho_{\text {expt }}^{\mathrm{W}_{3}}, \rho_{\text {expt }}^{\mathrm{C}_{4}}$, and $\rho_{\text {expt }}^{\mathrm{W}_{4}}$ and $86 \%$ for $\rho_{\text {expt }}^{\mathrm{GHZ}}$. Note that the estimated $l^{c}(\rho)$ of $\rho_{\text {expt }}^{\mathrm{GHZ}_{3}}, \rho_{\text {expt }}^{\mathrm{W}_{3}}, \rho_{\text {expt }}^{\mathrm{C}_{4}}$, and $\rho_{\text {expt }}^{\mathrm{GHZ}_{4}}$ is slightly more accurate than that of $\rho_{\text {expt }}^{\mathrm{W}_{4}}$ as shown in Figs. 3(a) and $3(\mathrm{c})$. The main reason is that the fidelity of prepared $\rho_{\text {expt }}^{\mathrm{W}_{4}}$ is slightly lower than that of the other four states as shown in Appendix B. The lower fidelity implies a larger distance between the prepared state and the target state, which indicates $\lambda \succ \boldsymbol{d} \vee\left(\wedge_{\boldsymbol{p} \in X} \boldsymbol{p}\right)$ so that $C_{\mathrm{RE}}(\rho)=S(\boldsymbol{d})-S(\boldsymbol{\lambda})>$ $l^{c}(\rho)=S(\boldsymbol{d})-S\left[\boldsymbol{d} \vee\left(\wedge_{\boldsymbol{p} \in X} \boldsymbol{p}\right)\right]$. However, it does not indicate that the lower fidelity always leads to the bigger gap between $C_{\mathrm{RE}}(\rho)$ and $l^{c}(\rho)$. If the prepared state is still a graph-diagonal state after considering the experimental imperfections, the estimated lower bound on such state is tight as well, i.e., $l^{c}(\rho)=C_{\mathrm{RE}}(\rho)$. In Appendix $\mathrm{C}$, we analyze our experimental imperfections and show how they affect the prepared states.

Finally, we estimate the upper bound $u^{c}$ of $\rho_{\text {expt }}^{\psi}$ by measuring the probability distribution $\boldsymbol{d}_{\text {expt }}^{\psi}$ on the basis of $Z^{\otimes n}$. The probability distributions $\boldsymbol{d}_{\text {expt }}^{\psi}$ are shown in Appendix B, by which we can calculate $u^{c}\left(\rho_{\text {expt }}^{\psi}\right)$ according to Eq. (8). The results of $u^{c}\left(\rho_{\text {expt }}^{\psi}\right)$ are shown with the blue dash-dotted line in Fig. 3(d). For all five states, we observe that $C_{\mathrm{RE}}\left(\rho_{\text {expt }}^{\psi}\right)$ lies within the range bounded by $l_{3,5}^{c}\left(\rho_{\text {expt }}^{\psi}\right)$ and $u^{c}\left(\rho_{\text {expt }}^{\psi}\right)$.

\section{CONCLUSION}

To conclude, we introduce an efficient and experimentally friendly estimation method for detecting coherence of multipartite states. We demonstrate that the coherence with high accuracy as well as high successful probability can be efficiently estimated with a few measurements for various multiqubit states. The procedure to obtain the lower bound is based on a few measurements of the stabilizing operators, similar to multipartite entanglement detection [60] and multipartite Bell inequalities [61]. This thus indicates that coherence and other resources can be inferred by the same set of measurements, which can further benefit our understanding of the connection between coherence and other resources $[15,30,31,33]$.

There are several open follow-up problems. The scheme to detect the lower bound of multipartite coherence is efficient and tight for graph-diagonal states as well as special types of quantum states that admit efficient classical representation (such as the W state). Exploring whether our scheme will work for a more general multiqubit quantum state is an interesting future direction. Besides, one may be concerned as to whether our scheme can be generalized to high-dimensional cases. For the multiqudit stabilizer state, its stabilizing operators constructed by generalized Pauli group are generally non-Hermitian [62-64], which cannot be observed directly in experiment. Recent studies indicate that expected values of non-Hermitian operators could be measured via weak measurements $[65,66]$. However, a definite answer may require rather sophisticated analysis. Finally, it is worth noting that a fidelity-based method was recently proposed to detect the lower bound of multipartite coherence via the convex roof construction [67]. Thus another open follow-up question is whether our scheme can be further generalized to other coherence measures while maintaining its appealing features of efficiency and simplicity.

\section{ACKNOWLEDGMENTS}

We are grateful to two anonymous referees for providing very useful comments on earlier versions of this manuscript. This work is supported by the National Natural 
Science Foundation of China (Grant No. 11974213 and No. 92065112), National Key R\&D Program of China (Grant No. 2019YFA0308200), and Shandong Provincial Natural Science Foundation (Grant No. ZR2019MA001 and No. ZR2020JQ05), Taishan Scholar of Shandong Province (Grant No. tsqn202103013) and Shandong University Multidisciplinary Research and Innovation Team of Young Scholars (Grant No. 2020QNQT).

\section{APPENDIX A: STABILIZING OPERATORS AND GRAPH-DIAGONAL BASIS}

\section{GHZ state}

For $n$-qubit GHZ states, the generators of $\mathcal{S}^{\mathrm{GHZ}}$ are

$$
\begin{aligned}
S_{1}^{\mathrm{GHZ}_{n}} & =\prod_{i=1}^{n} X^{(i)}, \\
S_{i}^{\mathrm{GHZ}_{n}} & =Z^{(i-1)} Z^{(i)} \quad \text { for } i=2,3, \ldots, n,
\end{aligned}
$$

and denoted as $\mathcal{S}^{\mathrm{GHZ}_{n}}=\left\langle S_{1}^{\mathrm{GHZ}_{n}}, S_{2}^{\left(\mathrm{GHZ}_{n}\right)}, \ldots, S_{n}^{\mathrm{GHZ}_{n}}\right\rangle$. For $\left|\mathrm{GHZ}_{3}\right\rangle$, the generators are $S_{1}^{\mathrm{GHZ}_{3}}=X X X, S_{2}^{\mathrm{GHZ}_{3}}=Z Z \mathbb{I}$, and $S_{3}^{\mathrm{GHZ}_{3}}=\mathbb{I} Z Z$. Then, all stabilizing operators can be obtained by multiplying them with each other, i.e.,

$$
\begin{aligned}
& S_{4}^{\mathrm{GHZ}_{3}}=S_{1}^{\mathrm{GHZ}_{3}} S_{2}^{\mathrm{GHZ}_{3}}=-Y Y X, \\
& S_{5}^{\mathrm{GHZ}_{3}}=S_{1}^{\mathrm{GHZ}_{3}} S_{3}^{\mathrm{GHZ}_{3}}=-X Y Y, \\
& S_{6}^{\mathrm{GHZ}_{3}}=S_{2}^{\mathrm{GHZ}^{3}} S_{3}^{\mathrm{GHZ}_{3}}=Z \mathbb{I} Z, \\
& S_{7}^{\mathrm{GHZ}_{3}}=S_{1}^{\mathrm{GHZ}_{3}} S_{2}^{\mathrm{GHZ}_{3}} S_{3}^{\mathrm{GHZ}_{3}}=-Y X Y, \\
& S_{8}^{\mathrm{GHZ}_{3}}=\mathbb{I} \mathbb{I} \mathbb{I} .
\end{aligned}
$$

The $\mathrm{GHZ}_{3}$-diagonal basis can be obtained from the computational basis $\left|k_{1} k_{2} k_{3}\right\rangle$ by performing a three-qubit unitary operation $U^{\mathrm{GHZ}_{3}}=\left(\mathrm{CNOT}_{12} \otimes \mathbb{I}_{3}\right) \cdot\left(\mathrm{CNOT}_{13} \otimes \mathbb{I}_{2}\right) \cdot\left(H_{1} \otimes\right.$ $\left.\mathbb{I}_{2} \otimes \mathbb{I}_{3}\right)$, where $H$ is the Hadamard operation and

$$
\text { CNOT }=\left(\begin{array}{llll}
1 & 0 & 0 & 0 \\
0 & 1 & 0 & 0 \\
0 & 0 & 0 & 1 \\
0 & 0 & 1 & 0
\end{array}\right)
$$

is the unitary matrix of the controlled-NOT (CNOT) operation. Eight $\mathrm{GHZ}_{3}$-diagonal bases are determined by $\left|\psi_{i}^{\mathrm{GHZ}_{3}}\right\rangle=$ $U^{\mathrm{GHZ}_{3}}\left|k_{1} k_{2} k_{3}\right\rangle$ and shown in Table I.

The generators of $\left|\mathrm{GHZ}_{4}\right\rangle$ are $S_{1}^{\mathrm{GHZ}_{4}}=X X X X, S_{2}^{\mathrm{GHZ}_{4}}=$ $Z Z \mathbb{I} \mathbb{I}, S_{3}^{\mathrm{GHZ}_{4}}=\mathbb{I} Z Z \mathbb{I}$, and $S_{4}^{\mathrm{GHZ}_{4}}=\mathbb{I} \mathbb{I} Z Z$. Other stabilizing

TABLE I. The $\mathrm{GHZ}_{3}$-diagonal bases and the corresponding expected values of generators $\left(\left\langle S_{1}^{\mathrm{GHZ}_{3}}\right\rangle,\left\langle S_{2}^{\mathrm{GHZ}_{3}}\right\rangle,\left\langle S_{3}^{\mathrm{GHZ}_{3}}\right\rangle\right)$.

\begin{tabular}{lcc}
\hline \hline$k_{1} k_{2} k_{3}$ & $\mid \psi_{i}^{\left.\mathrm{GHZ}_{3}\right\rangle}$ & $\left(\left\langle S_{1}^{\mathrm{GHZ}_{3}}\right\rangle,\left\langle S_{2}^{\mathrm{GHZ}_{3}}\right\rangle,\left\langle S_{3}^{\mathrm{GHZ}_{3}}\right\rangle\right)$ \\
\hline 000 & $(|000\rangle+|111\rangle) / \sqrt{2}$ & $(+1,+1,+1)$ \\
001 & $(|001\rangle+|110\rangle) / \sqrt{2}$ & $(+1,+1,-1)$ \\
010 & $(|010\rangle+|101\rangle) / \sqrt{2}$ & $(+1,-1,-1)$ \\
011 & $(|011\rangle+|100\rangle) / \sqrt{2}$ & $(+1,-1,+1)$ \\
100 & $(|000\rangle-|111\rangle) / \sqrt{2}$ & $(-1,+1,+1)$ \\
101 & $(|001\rangle-|110\rangle) / \sqrt{2}$ & $(-1,+1,-1)$ \\
110 & $(|010\rangle-|101\rangle) / \sqrt{2}$ & $(-1,-1,-1)$ \\
111 & $(|011\rangle-|100\rangle) / \sqrt{2}$ & $(-1,-1,+1)$ \\
\hline \hline
\end{tabular}

TABLE II. The $\mathrm{GHZ}_{4}$-diagonal bases and the corresponding expected values of generators $\left(\left\langle S_{1}^{\mathrm{GHZ}_{4}}\right\rangle,\left\langle S_{2}^{\mathrm{GHZ}_{4}}\right\rangle,\left\langle S_{3}^{\mathrm{GHZ}_{4}}\right\rangle,\left\langle S_{4}^{\mathrm{GHZ}_{4}}\right\rangle\right)$.

\begin{tabular}{lcc}
\hline \hline$k_{1} k_{2} k_{3} k_{4}$ & $\left|\psi_{i}^{\mathrm{GHZ}_{4}}\right\rangle$ & $\left(\left\langle S_{1}^{\mathrm{GHZ}_{4}}\right\rangle,\left\langle S_{2}^{\mathrm{GHZ}_{4}}\right\rangle,\left\langle S_{3}^{\mathrm{GHZ}_{4}}\right\rangle,\left\langle S_{4}^{\mathrm{GHZ}_{4}}\right\rangle\right)$ \\
\hline 0000 & $(|0000\rangle+|1111\rangle) / \sqrt{2}$ & $(+1,+1,+1,+1)$ \\
0001 & $(|0001\rangle+|1110\rangle) / \sqrt{2}$ & $(+1,+1,+1,-1)$ \\
0010 & $(|0010\rangle+|1101\rangle) / \sqrt{2}$ & $(+1,+1,-1,-1)$ \\
0011 & $(|0011\rangle+|1100\rangle) / \sqrt{2}$ & $(+1,+1,-1,+1)$ \\
0100 & $(|0100\rangle+|1011\rangle) / \sqrt{2}$ & $(+1,-1,-1,+1)$ \\
0101 & $(|0101\rangle+|1010\rangle) / \sqrt{2}$ & $(+1,-1,-1,-1)$ \\
0110 & $(|0110\rangle+|1001\rangle) / \sqrt{2}$ & $(+1,-1,+1,-1)$ \\
0111 & $(|0111\rangle+|1000\rangle) / \sqrt{2}$ & $(+1,-1,+1,+1)$ \\
1000 & $(|0000\rangle-|1111\rangle) / \sqrt{2}$ & $(-1,+1,+1,+1)$ \\
1001 & $(|0001\rangle-|1110\rangle) / \sqrt{2}$ & $(-1,+1,+1,-1)$ \\
1010 & $(|0010\rangle-|1101\rangle) / \sqrt{2}$ & $(-1,+1,-1,-1)$ \\
1011 & $(|0011\rangle-|1100\rangle) / \sqrt{2}$ & $(-1,+1,-1,+1)$ \\
1100 & $(|0100\rangle-|1011\rangle) / \sqrt{2}$ & $(-1,-1,-1,+1)$ \\
1101 & $(|0101\rangle-|1010\rangle) / \sqrt{2}$ & $(-1,-1,-1,-1)$ \\
1110 & $(|0110\rangle-|1001\rangle) / \sqrt{2}$ & $(-1,-1,+1,-1)$ \\
1111 & $(|0111\rangle-|1000\rangle) / \sqrt{2}$ & $(-1,-1,+1,+1)$ \\
\hline \hline
\end{tabular}

operators are

$$
\begin{aligned}
& S_{5}^{\mathrm{GHZ}_{4}}=-Y Y X X, \quad S_{6}^{\mathrm{GHZ}_{4}}=-X Y Y X, \\
& S_{7}^{\mathrm{GHZ}_{4}}=-X X Y Y, \quad S_{8}^{\mathrm{GHZ}_{4}}=Z \mathbb{I} Z \mathbb{I}, \\
& S_{9}^{\mathrm{GHZ}_{4}}=Z Z Z Z, \quad S_{10}^{\mathrm{GHZ}_{4}}=\mathbb{I} Z \mathbb{I} Z, \\
& S_{11}^{\mathrm{GHZ}_{4}}=-Y X Y X, \quad S_{12}^{\mathrm{GHZ}_{4}}=Y Y Y Y, \\
& S_{13}^{\mathrm{GHZ}_{4}}=-X Y X Y, \quad S_{14}^{\mathrm{GHZ}_{4}}=Z \mathbb{I} \mathbb{I} Z, \\
& S_{15}^{\mathrm{GHZ}_{4}}=-Y X X Y, \quad S_{16}^{\mathrm{GHZ}_{4}}=\mathbb{I} \mathbb{I} \mathbb{I} \mathbb{I} .
\end{aligned}
$$

The $\mathrm{GHZ}_{4}$-diagonal basis can be obtained from the computational basis $\left|k_{1} k_{2} k_{3} k_{4}\right\rangle$ by performing a four-qubit unitary operation $U^{\mathrm{GHZ}_{4}}=\left(\mathbb{I}_{1} \otimes H_{2} \otimes H_{3} \otimes H_{4}\right) \cdot\left(\mathrm{CZ}_{14}\right) \cdot\left(\mathrm{CZ}_{13}\right)$. $\left(\mathrm{CZ}_{12}\right) \cdot\left(H_{1} \otimes H_{2} \otimes H_{3} \otimes H_{4}\right)$, where

$$
\mathrm{CZ}=\left(\begin{array}{cccc}
1 & 0 & 0 & 0 \\
0 & 1 & 0 & 0 \\
0 & 0 & 1 & 0 \\
0 & 0 & 0 & -1
\end{array}\right)
$$

is the unitary matrix of the controlled- $Z$ (CZ) operation. Sixteen $\mathrm{GHZ}_{4}$-diagonal bases are determined by $\left|\psi_{i}^{\mathrm{GHZ}_{4}}\right\rangle=$ $U^{\mathrm{GHZ}_{4}}\left|k_{1} k_{2} k_{3} k_{4}\right\rangle$ and shown in Table II.

\section{Cluster state}

The generators of a $n$-qubit cluster state $\left|\widetilde{\mathrm{C}}_{n}\right\rangle$ are

$$
\begin{aligned}
S_{1}^{\widetilde{\mathrm{C}}_{n}} & =X^{(1)} Z^{(2)}, \\
S_{i}^{\widetilde{\mathrm{C}}_{n}} & =Z^{(i-1)} X^{(i)} Z^{(i-1)} \quad \text { for } i=2,3, \ldots, n-1, \\
S_{n}^{\widetilde{\mathrm{C}}_{n}} & =Z^{(n-1)} X^{(n)} .
\end{aligned}
$$

The generators of a four-qubit linear graph are $S_{1}^{\widetilde{\mathrm{C}}_{4}}=X Z \mathbb{I} \mathbb{I}$, $S_{2}^{\widetilde{\mathrm{C}}_{4}}=Z X Z \mathbb{I}, S_{3}^{\widetilde{\mathrm{C}}_{4}}=\mathbb{I} Z X Z$, and $S_{4}^{\widetilde{\mathrm{C}}_{4}}=\mathbb{I} \mathbb{I} Z X$, and the corresponding state is $\left|\widetilde{\mathrm{C}}_{4}\right\rangle=(|+\underset{\widetilde{\mathrm{C}}}{+00+\rangle}\rangle+|+01-\rangle+|-10+\rangle-$ $|-11-\rangle) / 2$. Note that $\left|\widetilde{\mathrm{C}}_{4}\right\rangle$ can be transformed to the 
TABLE III. The cluster-diagonal bases and the corresponding expected values of generators $\left(\left\langle S_{1}^{\mathrm{C}_{4}}\right\rangle,\left\langle S_{2}^{\mathrm{C}_{4}}\right\rangle,\left\langle S_{3}^{\mathrm{C}_{4}}\right\rangle,\left\langle S_{4}^{\mathrm{C}_{4}}\right\rangle\right)$

\begin{tabular}{lcc}
\hline \hline$k_{1} k_{2} k_{3} k_{4}$ & $\left|\psi_{i}^{\mathrm{C}_{4}}\right\rangle$ & $\left(\left\langle S_{1}^{\mathrm{C}_{4}}\right\rangle,\left\langle S_{2}^{\mathrm{C}_{4}}\right\rangle,\left\langle S_{3}^{\mathrm{C}_{4}}\right\rangle,\left\langle S_{4}^{\mathrm{C}_{4}}\right\rangle\right)$ \\
\hline 0000 & $\frac{|0000\rangle+|0011\rangle+|1100\rangle-|1111\rangle}{2}$ & $(+1,+1,+1,+1)$ \\
0001 & $\frac{|0001\rangle+|0010\rangle+|1101\rangle-|1110\rangle}{2}$ & $(+1,+1,+1,-1)$ \\
0010 & $\frac{|0000\rangle-|0011\rangle+|1100\rangle+|1111\rangle}{2}$ & $(+1,+1,-1,+1)$ \\
0011 & $\frac{|0001\rangle-|0010\rangle+|1101\rangle+|1110\rangle}{2}$ & $(+1,+1,-1,-1)$ \\
0100 & $\frac{|0100\rangle-|0111\rangle+|1000\rangle+|1011\rangle}{2}$ & $(-1,+1,+1,+1)$ \\
0101 & $\frac{|0101\rangle-|0110\rangle+|1001\rangle+|1010\rangle}{2}$ & $(-1,+1,+1,-1)$ \\
0110 & $\frac{|0100\rangle+|0111\rangle+|1000\rangle-|1011\rangle}{2}$ & $(-1,+1,-1,+1)$ \\
0111 & $\frac{|0101\rangle+|0110\rangle+|1001\rangle-|1010\rangle}{2}$ & $(-1,+1,-1,-1)$ \\
1000 & $\frac{|0000\rangle+|0011\rangle-|1100\rangle+|1111\rangle}{2}$ & $(+1,-1,+1,+1)$ \\
1001 & $\frac{|0001\rangle+|0010\rangle-|1101\rangle+|1110\rangle}{2}$ & $(+1,-1,+1,-1)$ \\
1010 & $\frac{|0000\rangle-|0011\rangle-|1100\rangle-|1111\rangle}{2}$ & $(+1,-1,-1,+1)$ \\
1011 & $\frac{|0001\rangle-|0010\rangle-|1101\rangle-|1110\rangle}{2}$ & $(+1,-1,-1,-1)$ \\
1100 & $\frac{|0100\rangle-|0111\rangle-|1000\rangle-|1011\rangle}{2}$ & $(-1,-1,+1,+1)$ \\
1101 & $\frac{|0101\rangle-|0110\rangle-|1001\rangle-|1010\rangle}{2}$ & $(-1,-1,+1,-1)$ \\
1110 & $\frac{|0100\rangle+|0111\rangle-|1000\rangle+|1011\rangle}{2}$ & $(-1,-1,-1,+1)$ \\
1111 & $\frac{|0101\rangle+|0110\rangle-|1001\rangle+|1010\rangle}{2}$ & $(-1,-1,-1,-1)$ \\
\hline \hline
\end{tabular}

common representation $\left|\mathrm{C}_{4}\right\rangle=(|0000\rangle+|0011\rangle+|1100\rangle-$

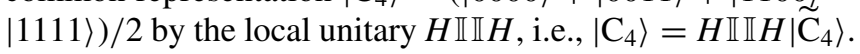
Accordingly, the generators of $\left|\mathrm{C}_{4}\right\rangle$ are transformed to $Z Z \mathbb{I} \mathbb{I}$, $X X Z \mathbb{I}, \mathbb{I} Z X X$, and $\mathbb{I} Z Z Z$. Other stabilizing operators are

$$
\begin{aligned}
& S_{5}^{\mathrm{C}_{4}}=-Y Y Z \mathbb{I}, \quad S_{6}^{\mathrm{C}_{4}}=Z \mathbb{I} X X, \\
& S_{7}^{\mathrm{C}_{4}}=Z Z Z Z, \quad S_{8}^{\mathrm{C}_{4}}=X Y Y X,
\end{aligned}
$$

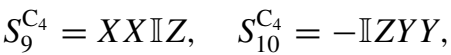

$$
\begin{aligned}
& S_{11}^{\mathrm{C}_{4}}=Y X Y X, \quad S_{12}^{\mathrm{C}_{4}}=-Y Y \mathbb{} Z,
\end{aligned}
$$

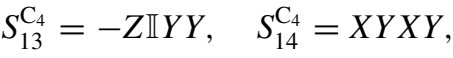

$$
\begin{aligned}
& S_{15}^{\mathrm{C}_{4}}=Y X X Y, \quad S_{16}^{\mathrm{C}_{4}}=\mathbb{I} \mathbb{I} \mathbb{I} .
\end{aligned}
$$

The cluster-diagonal basis can be obtained from the computational basis $\left|k_{1} k_{2} k_{3} k_{4}\right\rangle$ by performing a four-qubit unitary operation $U^{\mathrm{C}_{4}}=\left(\mathbb{I}_{1} \otimes \mathrm{CZ}_{23} \otimes \mathbb{I}_{4}\right) \cdot\left(\mathrm{CNOT}_{12} \otimes \mathrm{CNOT}_{34}\right)$. $\left(H_{1} \otimes \mathbb{I}_{2} \otimes H_{3} \otimes \mathbb{I}_{4}\right)$. Then, the cluster-diagonal bases are determined by $\left|\psi_{i}^{\mathrm{C}_{4}}\right\rangle=U^{\mathrm{C}_{4}}\left|k_{1} k_{2} k_{3} k_{4}\right\rangle$ and shown in Table III.

TABLE IV. The $\mathrm{W}_{3}$-diagonal bases and the corresponding expected values of generators $\left(\left\langle S_{1}^{\mathrm{W}_{3}}\right\rangle,\left\langle S_{2}^{\mathrm{W}_{3}}\right\rangle,\left\langle S_{3}^{\mathrm{W}_{3}}\right\rangle\right)$.

\begin{tabular}{lcc}
\hline \hline$k_{1} k_{2} k_{3}$ & $\left|\psi_{i}^{\mathrm{W}_{3}}\right\rangle$ & $\left(\left\langle S_{1}^{\mathrm{W}_{3}}\right\rangle,\left\langle S_{2}^{\mathrm{W}_{3}}\right\rangle,\left\langle S_{3}^{\mathrm{W}_{3}}\right\rangle\right)$ \\
\hline 000 & $(|001\rangle+|010\rangle+|100\rangle) / \sqrt{3}$ & $(+1,+1,+1)$ \\
001 & $(|000\rangle-|011\rangle+|101\rangle) / \sqrt{3}$ & $(+1,+1,-1)$ \\
010 & $(|000\rangle+|011\rangle-|110\rangle) / \sqrt{3}$ & $(+1,-1,+1)$ \\
011 & $(-|001\rangle+|010\rangle-|111\rangle) / \sqrt{3}$ & $(+1,-1,-1)$ \\
100 & $(|000\rangle-|101\rangle+|110\rangle) / \sqrt{3}$ & $(-1,+1,+1)$ \\
101 & $(|001\rangle-|100\rangle-|111\rangle) / \sqrt{3}$ & $(-1,+1,-1)$ \\
110 & $(-|010\rangle+|100\rangle-|111\rangle) / \sqrt{3}$ & $(-1,-1,+1)$ \\
111 & $(-|011\rangle-|101\rangle-|110\rangle) / \sqrt{3}$ & $(-1,-1,-1)$ \\
\hline \hline
\end{tabular}

TABLE V. The $\mathrm{W}_{4}$-diagonal bases and the corresponding ex-

\begin{tabular}{|c|c|c|}
\hline$k_{1} k_{2} k_{3} k_{4}$ & $\left|\psi_{i}^{\mathrm{W}_{4}}\right\rangle$ & $\left(\left\langle S_{1}^{\mathrm{W}_{4}}\right\rangle,\left\langle S_{2}^{\mathrm{W}_{4}}\right\rangle,\left\langle S_{3}^{\mathrm{W}_{4}}\right\rangle,\left\langle S_{4}^{\mathrm{W}_{4}}\right\rangle\right)$ \\
\hline 0000 & $\frac{|0001\rangle+|0010\rangle+|0100\rangle+|1000\rangle}{2}$ & $(+1,+1,+1,+1)$ \\
\hline 0001 & $\frac{|0000\rangle+|0011\rangle+|0101\rangle+|1001\rangle}{2}$ & $(-1,+1,+1,+1)$ \\
\hline 0010 & $\frac{|0000\rangle-|0011\rangle+|0110\rangle+|1010\rangle}{2}$ & $(+1,-1,+1,+1)$ \\
\hline 0011 & $\frac{|0001\rangle-|0010\rangle+|0111\rangle+|1011\rangle}{2}$ & $(-1,-1,+1,+1)$ \\
\hline 0100 & $\frac{|0000\rangle-|0101\rangle^{2}-|0110\rangle+|1100\rangle}{2}$ & $(+1,+1,-1,+1)$ \\
\hline 0101 & $\frac{|0001\rangle-|0100\rangle-|0111\rangle+|1101\rangle}{2}$ & $(-1,+1,-1,+1)$ \\
\hline 0110 & $\frac{|0010\rangle-|0100\rangle^{2}+|0111\rangle+|1110\rangle}{2}$ & $(1,-1,-1,+1)$ \\
\hline 0111 & $\frac{|0011\rangle-|0101\rangle+|0110\rangle+|1111\rangle}{2}$ & $(-1,-1,-1,+1)$ \\
\hline 1000 & $\frac{|0000\rangle-|1001\rangle-|1010\rangle-|1100\rangle}{2}$ & $(+1,+1,+1,-1)$ \\
\hline 1001 & $\frac{|0001\rangle-|1000\rangle-|1011\rangle-|1101\rangle}{2}$ & $(-1,+1,+1,-1)$ \\
\hline 1010 & $\frac{|0010\rangle-|1000\rangle+|1011\rangle-|1110\rangle}{2}$ & $(+1,-1,+1,-1)$ \\
\hline 1011 & $\frac{|0011\rangle-|1001\rangle+|1010\rangle-|1111\rangle}{2}$ & $(-1,-1,+1,-1)$ \\
\hline 1100 & $\frac{|0100\rangle-|1000\rangle+|1101\rangle+|1110\rangle}{2}$ & $(+1,+1,-1,-1)$ \\
\hline 1101 & $\frac{|0101\rangle-|1001\rangle^{2}+|1100\rangle+|1111\rangle}{2}$ & $(-1,+1,-1,-1)$ \\
\hline 1110 & $\frac{|0110\rangle-|1010\rangle^{2}+|1100\rangle-|1111\rangle}{2}$ & $(+1,-1,-1,-1)$ \\
\hline 1111 & $\frac{|0111\rangle-|1011\rangle+|1101\rangle-|1110\rangle}{2}$ & $(-1,-1,-1,-1)$ \\
\hline
\end{tabular}
pected values of generators $\left(\left\langle S_{1}^{\mathrm{W}_{4}}\right\rangle,\left\langle S_{2}^{\mathrm{W}_{4}}\right\rangle,\left\langle S_{3}^{\mathrm{W}_{4}}\right\rangle,\left\langle S_{4}^{\mathrm{W}_{4}}\right\rangle\right)$.

\section{W state}

$\left|\mathrm{W}_{3}\right\rangle$ can be transformed from $|000\rangle$ by the unitary $U^{\mathrm{W}_{3}}=$ $(X Z \mathbb{I}+\mathbb{I} X Z+Z \mathbb{I} X) / \sqrt{3}$, i.e., $\left|\mathrm{W}_{3}\right\rangle=U^{\mathrm{W}_{3}}|000\rangle[60]$. Thus the generators of $\left|\mathrm{W}_{3}\right\rangle$ are derived by

$$
\begin{aligned}
& S_{1}^{\mathrm{W}_{3}}=U^{\mathrm{W}_{3}} Z \mathbb{I} \mathbb{I} U^{\mathrm{W}_{3}^{\dagger}}=\frac{1}{3}(Z \mathbb{I} \mathbb{I}+2 Y Y Z+2 X Z X), \\
& S_{2}^{\mathrm{W}_{3}}=U^{\mathrm{W}_{3}} \mathbb{I} Z \mathbb{I} U^{\mathrm{W}_{3}^{\dagger}}=\frac{1}{3}(\mathbb{I} Z \mathbb{I}+2 Z Y Y+2 X X Z), \\
& S_{3}^{\mathrm{W}_{3}}=U^{\mathrm{W}_{3}} \mathbb{I} \mathbb{I} Z U^{\mathrm{W}_{3}^{\dagger}}=\frac{1}{3}(\mathbb{I} \mathbb{I} Z+2 Y Z Y+2 Z X X) .
\end{aligned}
$$

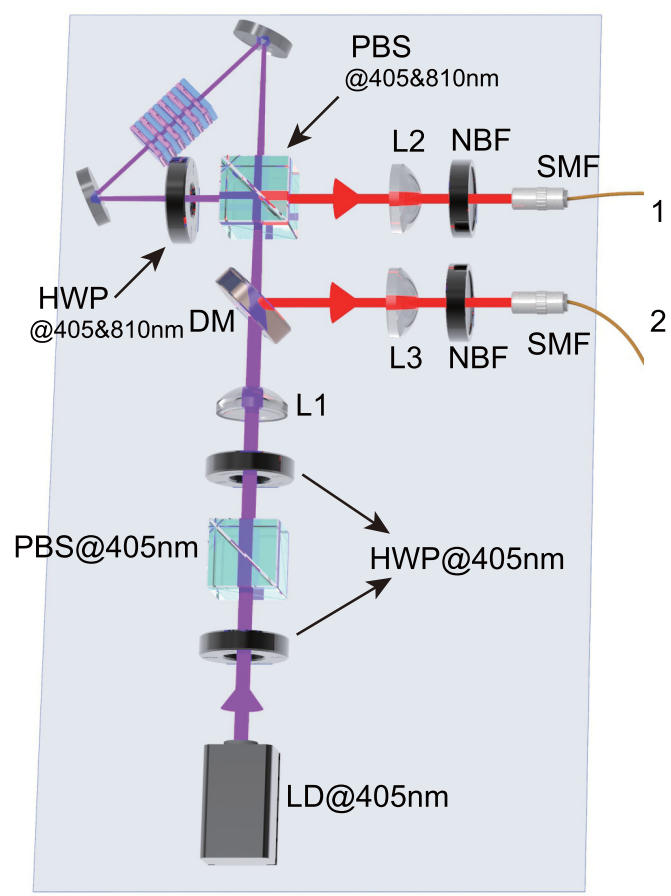

FIG. 4. Illustration of experimental setup to generate a polarization-entangled photon pair. $\mathrm{LD}$, laser diode. 
Other stabilizing operators are

$$
\begin{aligned}
S_{4}^{\mathrm{W}_{3}} & =\frac{1}{3}(2 X \mathbb{I} X+2 \mathbb{I} Y Y-Z Z \mathbb{I}), \\
S_{5}^{\mathrm{W}_{3}} & =\frac{1}{3}(2 \mathbb{I} X X+2 Y Y \mathbb{I}-Z \mathbb{I} Z), \\
S_{6}^{\mathrm{W}_{3}} & =\frac{1}{3}(2 X X \mathbb{I}+2 Y \mathbb{I} Y-\mathbb{I} Z Z), \\
S_{7}^{\mathrm{W}_{3}} & =-Z Z Z, \quad S_{8}^{\mathrm{W}_{3}}=\mathbb{I} \mathbb{I} \mathbb{I} .
\end{aligned}
$$

The $\mathrm{W}_{3}$-diagonal bases are shown in Table IV.

Similarly, we can find a possible unitary operator $U^{\mathrm{W}_{4}}=$ $(Z Z Z X+Z Z X \mathbb{I}+Z X \mathbb{I} \mathbb{I}+X \mathbb{I} \mathbb{I}) / 2$ to generate $\left|\mathrm{W}_{4}\right\rangle$ from $|0000\rangle$. Thus the generators of $\left|\mathrm{W}_{4}\right\rangle$ can be obtained, i.e.,

$$
\begin{aligned}
& S_{1}^{\mathrm{W}_{4}}=\frac{1}{2}(Y Z Z Y+\mathbb{I} Y Z Y+\mathbb{I} Y Y+\mathbb{I} \mathbb{I} Z), \\
& S_{2}^{\mathrm{W}_{4}}=\frac{1}{2}(Y Z Y \mathbb{I}+\mathbb{I} Y Y \mathbb{I}+\mathbb{I} \mathbb{I} Z \mathbb{I}+\mathbb{I} X X), \\
& S_{3}^{\mathrm{W}_{4}}=\frac{1}{2}(Y Y \mathbb{I}+\mathbb{I} Z \mathbb{I} \mathbb{I}+\mathbb{I} X Z X+\mathbb{I} X X \mathbb{I}), \\
& S_{4}^{\mathrm{W}_{4}}=\frac{1}{2}(Z \mathbb{I} \mathbb{I}+X Z Z X+X Z X \mathbb{I}+X X \mathbb{I}),
\end{aligned}
$$

and the other stabilizing operators are

$$
\begin{aligned}
S_{5}^{\mathrm{W}_{4}} & =\frac{1}{2}(Y Z Y Z+Y Z \mathbb{I}+\mathbb{I} Y Y Z+\mathbb{I} Y \mathbb{I} Y), \\
S_{6}^{\mathrm{W}_{4}} & =\frac{1}{2}(Y Y \mathbb{I} Z+Y \mathbb{I} Z Y+\mathbb{I} Z Y Y+\mathbb{I} X X Z), \\
S_{7}^{\mathrm{W}_{4}} & =\frac{1}{2}(Z Y Z Y+Z \mathbb{I} Y Y+X Z X Z+X X \mathbb{I} Z), \\
S_{8}^{\mathrm{W}_{4}} & =\frac{1}{2}(Y Y Z \mathbb{I}+Y \mathbb{I} Y \mathbb{I}+\mathbb{I} Z X X+\mathbb{I} X \mathbb{I} X), \\
S_{9}^{\mathrm{W}_{4}} & =\frac{1}{2}(Z Y Y \mathbb{I}+Z \mathbb{I} X X+X Z \mathbb{I} X+X X Z \mathbb{I}),
\end{aligned}
$$

$$
\begin{aligned}
S_{10}^{\mathrm{W}_{4}} & =\frac{1}{2}(Z X Z X+Z X X \mathbb{I}+X \mathbb{I} Z X+X \mathbb{I} X \mathbb{I}), \\
S_{11}^{\mathrm{W}_{4}} & =\frac{1}{2}(Y Y Z Z+Y \mathbb{I} Z+Y \mathbb{I} Y-\mathbb{I} Z Z Z), \\
S_{12}^{\mathrm{W}_{4}} & =\frac{1}{2}(Z Y Y Z+Z Y \mathbb{I} Y-Z \mathbb{I} Z Z+X X Z Z), \\
S_{13}^{\mathrm{W}_{4}} & =\frac{1}{2}(Z Z Y Y-Z Z \mathbb{I}+Z X X Z+X \mathbb{I} X Z), \\
S_{14}^{\mathrm{W}_{4}} & =\frac{1}{2}(-Z Z Z \mathbb{I}+Z Z X X+Z X \mathbb{I} Z+X \mathbb{I} X), \\
S_{15}^{\mathrm{W}_{4}} & =-Z Z Z Z, \quad S_{16}^{\mathrm{W}_{4}}=\mathbb{I} \mathbb{I} \mathbb{I} .
\end{aligned}
$$

The $\mathrm{W}_{4}$-diagonal bases are shown in Table $\mathrm{V}$.

\section{APPENDIX B: DETAILS OF EXPERIMENTAL REALIZATIONS AND RESULTS}

In this Appendix, we provide further details about our experimental setup. It would be useful to bear in mind the following:

(i) A half-wave plate (HWP) at $\theta$ performs the unitary transformation $U_{\mathrm{HWP}}=\cos 2 \theta(|H\rangle\langle H|-| V\rangle\langle V|)+$ $\sin 2 \theta(|H\rangle\langle V|+| V\rangle\langle H|)$ on a polarization state, where $\theta$ is the angle between the fast axis of the HWP and vertical polarization.

(ii) A beam displacer (BD) transmits a vertically polarized photon but deviates a horizontally polarized one.

(iii) A polarized beam splitter (PBS) transmits a horizontally polarized photon but reflects a vertically polarized one.

(iv) A quarter-wave plate (QWP) at $\theta$ performs the unitary transformation $U_{\mathrm{QWP}}=\frac{1}{\sqrt{2}}\left[\mathbb{I}_{2}+i \cos 2 \theta(|H\rangle\langle H|-| V\rangle\langle V|)+\right.$

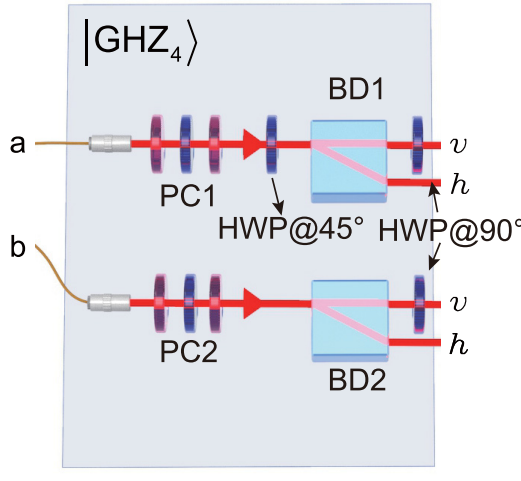

(a)

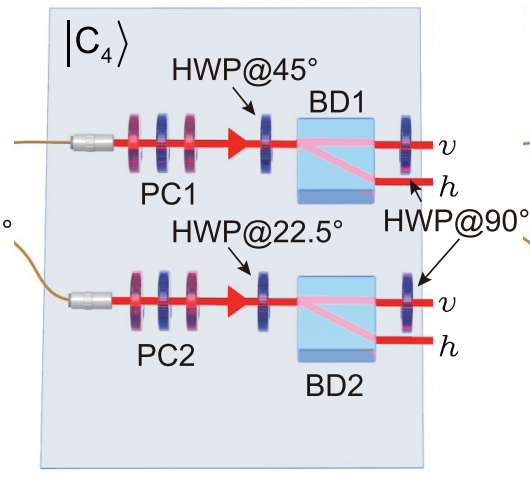

(b)

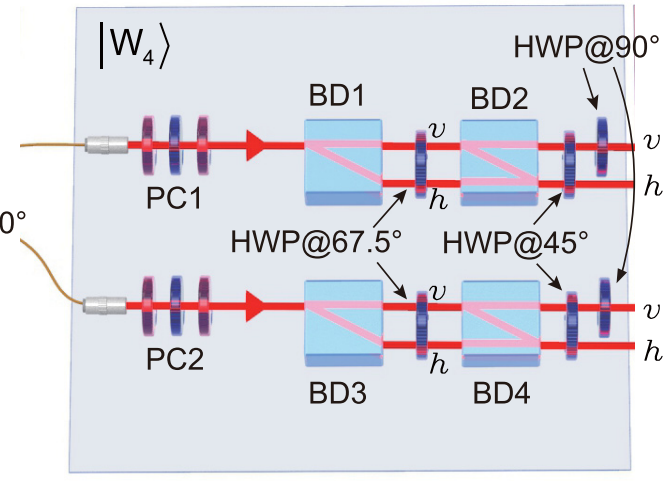

(c)

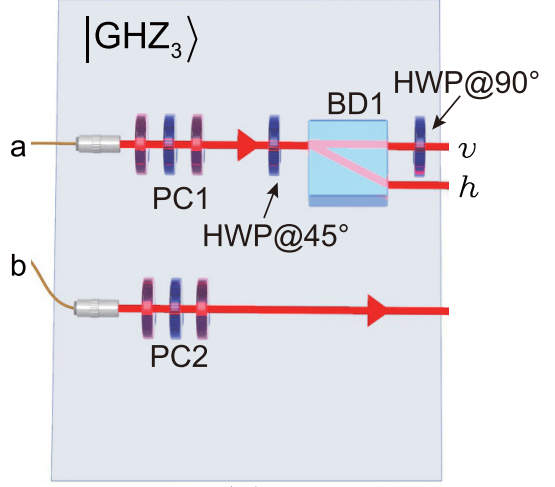

(d)

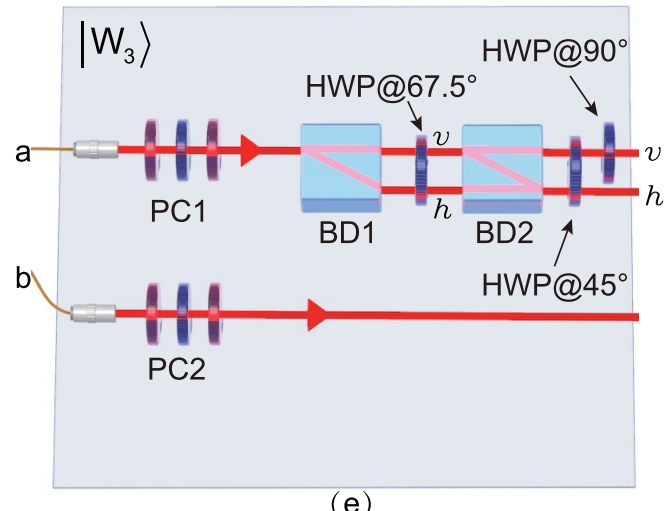

(e)

FIG. 5. Illustration of the experimental setups to generate a multiqubit state: (a) the four-qubit GHZ state $\left|\mathrm{GHZ}_{4}\right\rangle$, (b) the four-qubit cluster state $\left|\mathrm{C}_{4}\right\rangle$, (c) the four-qubit W state $\left|\mathrm{W}_{4}\right\rangle$, (d) the three-qubit GHZ state $\left|\mathrm{GHZ}_{3}\right\rangle$, and (e) the three-qubit W state $\left|\mathrm{W}_{3}\right\rangle$. 


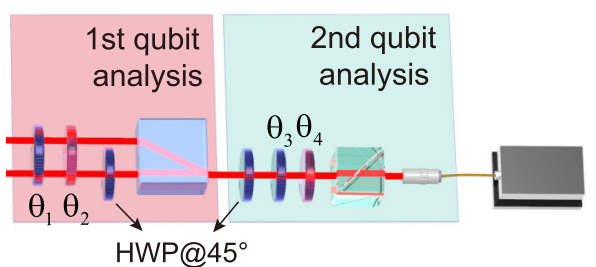

FIG. 6. Experimental setups of measurement on a photon encoded in the polarization DOF and path DOF.

$i \sin 2 \theta(|H\rangle\langle V|+| V\rangle\langle H|)]$, where $\mathbb{I}_{2}=|H\rangle\langle H|+| V\rangle\langle V|$ and $\theta$ is the angle between the fast axis of the QWP and vertical polarization, on a polarization state.

\section{Polarization-entangled photon source}

As shown in Fig. 4, the power of the pump light can be adjusted by a HWP and a PBS. After the PBS, horizontal polarization $\left|H_{p}\right\rangle$ is rotated to $\left|+_{p}\right\rangle=\frac{1}{\sqrt{2}}\left(\left|H_{p}\right\rangle+\left|V_{p}\right\rangle\right)$ by a HWP set at $22.5^{\circ}$. The pump beam is focused into a PPKTP crystal with a beam waist of $74 \mu \mathrm{m}$ by two lenses $\mathrm{L}_{1}$, whose focal lengths are 75 and $125 \mathrm{~mm}$, respectively. The PPKTP crystal, with dimensions of $10 \times 2 \times 1 \mathrm{~mm}$ (length $\times$ width $\times$ thickness) and poling period of $\Lambda=10.025 \mu \mathrm{m}$, is held in a home-built copper oven, and the temperature is controlled by a homemade temperature controller, which is set at $29{ }^{\circ} \mathrm{C}$ to realize the optimum type-II phase matching at $810 \mathrm{~nm}$. Then, the pump beam is split by a dual-wavelength PBS and coherently pumps PPKTP in the clockwise and counterclockwise direction, respectively. The clockwise and counterclockwise photons are recombined at the dual-wavelength PBS to generate polarization-entangled photons with an ideal form of $\left|\Psi_{13}^{+}\right\rangle=\frac{1}{\sqrt{2}}\left(\left|H_{1} V_{3}\right\rangle+\left|V_{1} H_{3}\right\rangle\right)$. Two photons are filtered by narrowband filters (NBFs) with full width at half maximum (FWHM) of $3 \mathrm{~nm}$ and coupled into single-mode fibers (SMFs) by lenses of focal length $200 \mathrm{~mm}\left(\mathrm{~L}_{2}\right.$ and $\left.\mathrm{L}_{3}\right)$ and objective lenses (not shown in Fig. 4).

\section{Experimental setups to generate a multiqubit state}

We extend a photon to its path degree of freedom (DOF) using a BD with length of $28.3 \mathrm{~mm}$ and clear aperture of $10 \times 10 \mathrm{~mm}$. The BD transmits vertical polarization and deviates horizontal polarization by $3 \mathrm{~mm}$. Specifically, the experimental setups to generate the five multiqubit states $\left|\mathrm{GHZ}_{4}\right\rangle,\left|\mathrm{C}_{4}\right\rangle,\left|\mathrm{W}_{4}\right\rangle,\left|\mathrm{GHZ}_{3}\right\rangle$, and $\left|\mathrm{W}_{3}\right\rangle$ are shown in Figs. 5(a)-5(e), respectively. The step-by-step calculations are shown in Eqs. (B1)-(B3).

$$
\begin{aligned}
& \left|\Psi_{a b}^{+}\right\rangle=\frac{1}{\sqrt{2}}\left(\left|H_{1} V_{3}\right\rangle+\left|V_{1} H_{3}\right\rangle\right) \underset{\text { on path of photon } a}{\stackrel{\text { HWP at } 45^{\circ}}{\longrightarrow}} \frac{1}{\sqrt{2}}\left(\left|V_{1} V_{3}\right\rangle+\left|H_{1} H_{3}\right\rangle\right) \stackrel{\text { BD1 }}{\longrightarrow} \frac{1}{\sqrt{2}}\left(\left|V_{1}\right\rangle\left|v_{2}\right\rangle\left|V_{3}\right\rangle+\left|H_{1}\right\rangle\left|h_{2}\right\rangle\left|H_{3}\right\rangle\right)=\left|\mathrm{GHZ}_{3}\right\rangle \\
& \stackrel{\mathrm{BD} 2}{\longrightarrow} \frac{1}{\sqrt{2}}\left(\left|V_{1}\right\rangle\left|v_{2}\right\rangle\left|V_{3}\right\rangle\left|v_{4}\right\rangle+\left|H_{1}\right\rangle\left|h_{2}\right\rangle\left|H_{3}\right\rangle\left|h_{4}\right\rangle\right)=\left|\mathrm{GHZ}_{4}\right\rangle, \\
& \left|\Psi_{a b}^{+}\right\rangle=\frac{1}{\sqrt{2}}\left(\left|H_{1} V_{3}\right\rangle+\left|V_{1} H_{3}\right\rangle\right) \underset{\text { on path of photon } a}{\stackrel{\text { HWP at } 45^{\circ}}{\longrightarrow}} \frac{1}{\sqrt{2}}\left(\left|V_{1} V_{3}\right\rangle+\left|H_{1} H_{3}\right\rangle\right) \underset{\text { on path of photon } b}{\stackrel{\text { HWP at 22.5० }}{\longrightarrow}} \frac{1}{2}\left(\left|V_{1} H_{3}\right\rangle-\left|V_{1} V_{3}\right\rangle+\left|H_{1} H_{3}\right\rangle+\left|H_{1} V_{3}\right\rangle\right) \\
& \left.\stackrel{\mathrm{BD} 1, \mathrm{BD} 2}{\longrightarrow} \frac{1}{2}\left(\left|V_{1}\right\rangle\left|v_{2}\right\rangle\left|H_{3}\right\rangle\left|h_{4}\right\rangle-\left|V_{1}\right\rangle\left|v_{2}\right\rangle\left|V_{3}\right\rangle\left|v_{4}\right\rangle+\left|H_{1}\right\rangle\left|h_{2}\right\rangle\left|H_{3}\right\rangle\right)\left|h_{4}\right\rangle+\left|H_{1}\right\rangle\left|h_{2}\right\rangle\left|V_{3}\right\rangle\left|v_{4}\right\rangle\right)=\left|\mathrm{C}_{4}\right\rangle, \\
& \left|\Psi_{a b}^{+}\right\rangle=\frac{1}{\sqrt{2}}\left(\left|H_{1} V_{3}\right\rangle+\left|V_{1} H_{3}\right\rangle\right) \stackrel{\text { BD1 }}{\longrightarrow} \frac{1}{\sqrt{2}}\left(\left|H_{1}\right\rangle\left|h_{2}\right\rangle\left|V_{3}\right\rangle+\left|V_{1}\right\rangle\left|v_{2}\right\rangle\left|H_{3}\right\rangle\right) \\
& \underset{\text { on path } h \text { and } v \text { of photon } a}{\longrightarrow} \frac{1}{2}\left[\left(-\left|H_{1}\right\rangle+\left|V_{1}\right\rangle\right)\left|h_{2}\right\rangle\left|V_{3}\right\rangle+\left(\left|H_{1}\right\rangle+\left|V_{1}\right\rangle\right)\left|v_{2}\right\rangle\left|H_{3}\right\rangle\right] \\
& \stackrel{\mathrm{BD} 2}{\longrightarrow} \frac{1}{\sqrt{3}}\left(\left|V_{1}\right\rangle\left|h_{2}\right\rangle\left|V_{3}\right\rangle+\left|H_{1}\right\rangle\left|h_{2}\right\rangle\left|H_{3}\right\rangle+\left|V_{1}\right\rangle\left|v_{2}\right\rangle\left|H_{3}\right\rangle\right) \\
& \underset{\text { on path } h \text { and } v \text { of photon } a}{\longrightarrow} \frac{1}{\sqrt{3}}\left(\left|H_{1}\right\rangle\left|h_{2}\right\rangle\left|V_{3}\right\rangle+\left|V_{1}\right\rangle\left|h_{2}\right\rangle\left|H_{3}\right\rangle+\left|H_{1}\right\rangle\left|v_{2}\right\rangle\left|H_{3}\right\rangle\right)=\left|\mathrm{W}_{3}\right\rangle \\
& \stackrel{\mathrm{BD} 3}{\longrightarrow} \frac{1}{\sqrt{3}}\left(\left|H_{1}\right\rangle\left|h_{2}\right\rangle\left|V_{3}\right\rangle\left|v_{4}\right\rangle+\left|V_{1}\right\rangle\left|h_{2}\right\rangle\left|H_{3}\right\rangle\left|h_{4}\right\rangle+\left|H_{1}\right\rangle\left|v_{2}\right\rangle\left|H_{3}\right\rangle\left|h_{4}\right\rangle\right) \\
& \underset{\text { on path } h \text { and } v \text { of photon } b}{\stackrel{\text { HWP at } 675^{\circ}}{\longrightarrow}} \frac{1}{\sqrt{6}}\left[\left|H_{1}\right\rangle\left|h_{2}\right\rangle\left(\left|H_{3}\right\rangle+\left|V_{3}\right\rangle\right)\left|v_{4}\right\rangle+\left|V_{1}\right\rangle\left|h_{2}\right\rangle\left(-\left|H_{3}\right\rangle+\left|V_{3}\right\rangle\right)\left|h_{4}\right\rangle+\left|H_{1}\right\rangle\left|v_{2}\right\rangle\left(-\left|H_{3}\right\rangle+\left|V_{3}\right\rangle\right)\left|h_{4}\right\rangle\right] \\
& \stackrel{\mathrm{BD} 4}{\longrightarrow} \frac{1}{2}\left(\left|H_{1}\right\rangle\left|h_{2}\right\rangle\left|H_{3}\right\rangle\left|h_{4}\right\rangle+\left|H_{1}\right\rangle\left|h_{2}\right\rangle\left|V_{3}\right\rangle\left|v_{4}\right\rangle+\left|V_{1}\right\rangle\left|h_{2}\right\rangle\left|V_{3}\right\rangle\left|h_{4}\right\rangle+\left|H_{1}\right\rangle\left|v_{2}\right\rangle\left|V_{3}\right\rangle\left|h_{4}\right\rangle\right)
\end{aligned}
$$

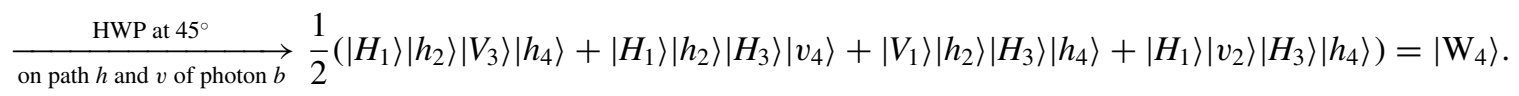


(a)
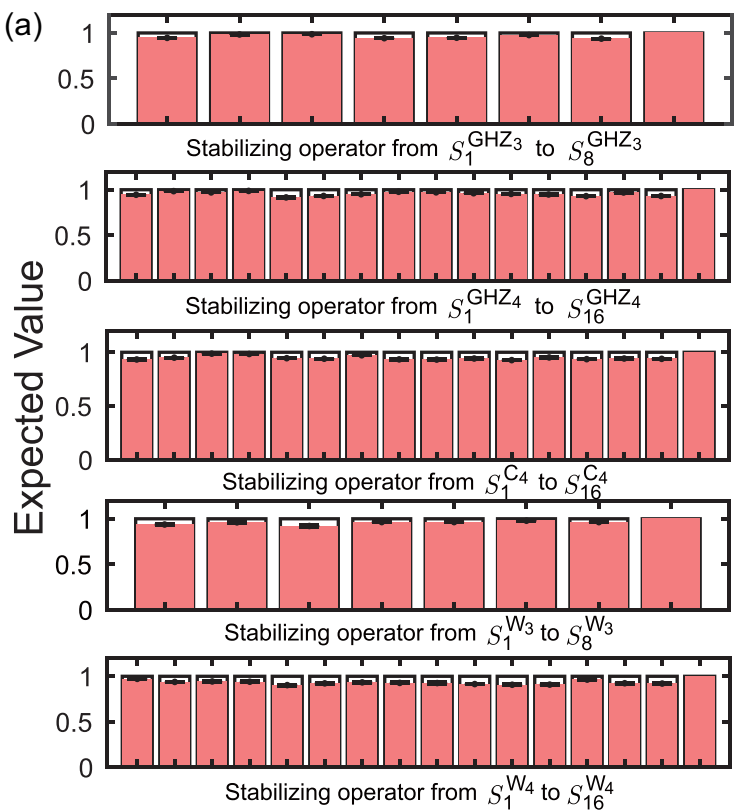

(b)
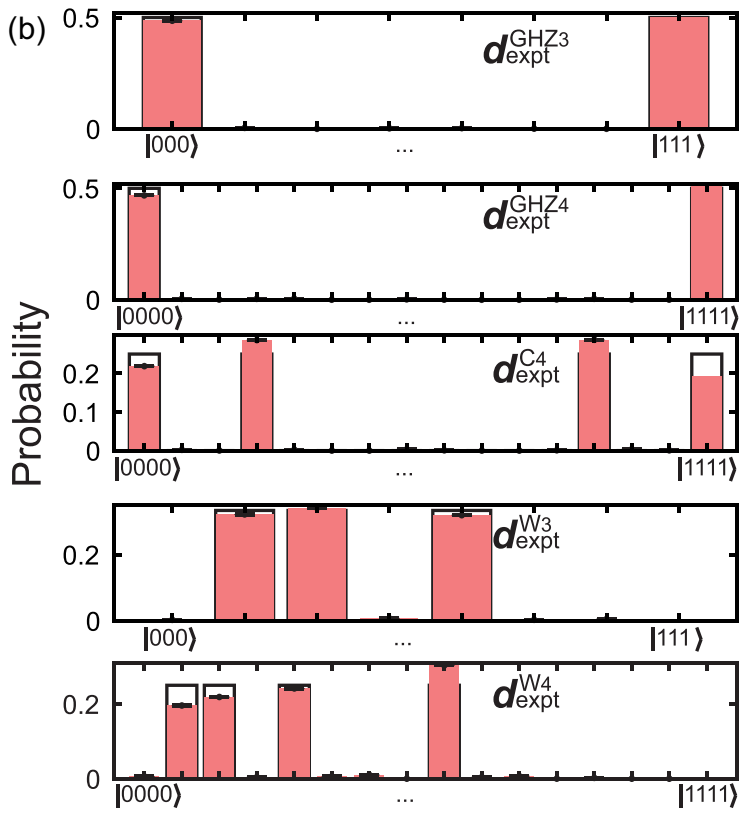

FIG. 7. Experimental results of (a) expected values of $\left\langle S_{i}^{\psi}\right\rangle$ and (b) probabilities of $\boldsymbol{d}_{\text {expt }}^{\psi}$ for states $\rho_{\text {expt }}^{\mathrm{GHZ}_{3}}, \rho_{\text {expt }}^{\mathrm{GHZ}}, \rho_{\text {expt }}^{\mathrm{C}_{4}}, \rho_{\text {expt }}^{\mathrm{W}_{3}}$, and $\rho_{\text {expt }}^{\mathrm{W}_{4}}$, respectively. The black grids represent the values for ideal states.

\section{Measurement and quantum state tomography}

If the photon is encoded in either the polarization DOF or the path DOF, we first perform the measurement on the polarization DOF and then on the path DOF. As illustrated in Fig. 6, the measurement basis of a qubit on the polarization DOF $\alpha|H\rangle+\beta|V\rangle$ is determined using the HWP at $\theta_{1}$ and the QWP at $\theta_{2}$. The measurement basis of a qubit on the path DOF $\gamma|h\rangle+\delta|v\rangle$ is determined using the HWP at $\theta_{3}$ and the QWP at $\theta_{4}$. Finally, a PBS is applied before the photon arrives at the detector. With this setting, the measurement on the basis $(\alpha|H\rangle+\beta|V\rangle) \otimes(\gamma|h\rangle+\delta|v\rangle)$ is achieved. The specific calculations are shown in eq. (B4). If the photon is only encoded in the polarization DOF, the measurement on the basis $\alpha|H\rangle+\beta|V\rangle$ is implemented by a QWP, HWP, and PBS.

$$
\begin{aligned}
& (\alpha|H\rangle+\beta|V\rangle) \otimes(\gamma|h\rangle+\delta|v\rangle) \\
& \underset{\text { QWP at } \theta_{2}}{\longrightarrow}|H\rangle \otimes(\gamma|h\rangle+\delta|v\rangle) \\
& \stackrel{\text { HWP at } 45^{\circ}}{\longrightarrow} \gamma|V\rangle|h\rangle+\delta|H\rangle|v\rangle \\
& \stackrel{\text { on path } h}{\longrightarrow} \gamma|V\rangle|h\rangle+\delta|H\rangle|h\rangle
\end{aligned}
$$

$$
\begin{aligned}
& \stackrel{\text { HWP at } 45^{\circ}}{\longrightarrow}(\gamma|H\rangle+\delta|V\rangle)|h\rangle \\
& \underset{\text { QWP at } \theta_{4}}{\stackrel{\text { HWP at } \theta_{3}}{\longrightarrow}}|H\rangle|h\rangle \\
& \stackrel{\text { PBS }}{\longrightarrow}|H\rangle|h\rangle \text {. }
\end{aligned}
$$

With this experimental setting, we can perform the measurement on an arbitrary basis. The experimental results of $\left\langle S_{i}^{\psi}\right\rangle$ and $\boldsymbol{d}_{\text {expt }}^{\psi}$ are shown in Figs. 7(a) and 7(b), respectively. Moreover, we reconstruct experimentally generated states $\rho_{\text {expt }}^{\mathrm{GHZ}_{4}}, \rho_{\text {expt }}^{\mathrm{C}_{4}}, \rho_{\text {expt }}^{\mathrm{W}_{4}}, \rho_{\text {expt }}^{\mathrm{GHZ}_{3}}$, and $\rho_{\text {expt }}^{\mathrm{W}_{3}}$ by quantum state tomography [48]. The results are shown in Fig. 8, from which we calculate the fidelity $F^{\psi}=$ $\operatorname{tr}\left(\rho_{\text {expt }}^{\psi}|\psi\rangle\langle\psi|\right)$. We observe that $F^{\mathrm{GHZ}_{3}}=0.9643 \pm 0.0003$, $F^{\mathrm{W}_{3}}=0.9589 \pm 0.0005, F^{\mathrm{GHZ}_{4}}=0.9571 \pm 0.0003, F^{\mathrm{C}_{4}}=$ $0.9497 \pm 0.0002$, and $F^{\mathrm{W}_{4}}=0.915 \pm 0.001$. Also, the relative entropy of coherence $C_{\mathrm{RE}}\left(\rho_{\text {expt }}^{\psi}\right)$ of $\rho_{\text {expt }}^{\psi}$ can be calculated by $C_{\mathrm{RE}}(\rho)=S_{\mathrm{VN}}\left(\rho_{d}\right)-S_{\mathrm{VN}}(\rho)$, according to which we obtain $C_{\mathrm{RE}}\left(\rho_{\mathrm{expt}}^{\mathrm{GHZ}_{3}}\right)=0.875 \pm 0.002, C_{\mathrm{RE}}\left(\rho_{\mathrm{expt}}^{\mathrm{W}_{3}}\right)=1.479 \pm$ $0.004, C_{\mathrm{RE}}\left(\rho_{\mathrm{expt}}^{\mathrm{GHZ}_{4}}\right)=0.906 \pm 0.002, C_{\mathrm{RE}}\left(\rho_{\mathrm{expt}}^{\mathrm{C}_{4}}\right)=1.806 \pm$ 0.002 , and $C_{\mathrm{RE}}\left(\rho_{\text {expt }}^{\mathrm{W}_{4}}\right)=1.964 \pm 0.003$.

\section{APPENDIX C: EXPERIMENTAL IMPERFECTIONS}

In our experiment, the main imperfection of prepared multiqubit states comes from the imperfection of the polarizationentangled photon pair, which is caused by the mode mismatch of overlapping lights on the PBS in Fig. 4. The noisy state can be described by a dephasing channel acting on ideal $\left|\Phi^{+}\right\rangle=(|H H\rangle+|V V\rangle) / \sqrt{2}$ by

$$
\rho^{\Psi^{+}}=\mathcal{E}\left(\left|\Phi^{+}\right\rangle\left\langle\Phi^{+}\right|\right)=(1-\mu)\left|\Phi^{+}\right\rangle\left\langle\Phi^{+}\right|+\frac{\mu}{2}(|H H\rangle\langle H H|+| V V\rangle\langle V V|) .
$$

With such a noisy state and following the procedure of generating a multiqubit state in Appendix B 2, we calculate the noisy multiqubit states in the computational basis (CB)

$$
\rho_{\mathrm{CB}}^{\mathrm{GHZ}_{3}}=(1-\mu)\left|\mathrm{GHZ}_{3}\right\rangle\left\langle\mathrm{GHZ}_{3}\right|+\frac{\mu}{2}(|000\rangle\langle 000|+| 111\rangle\langle 111|),
$$




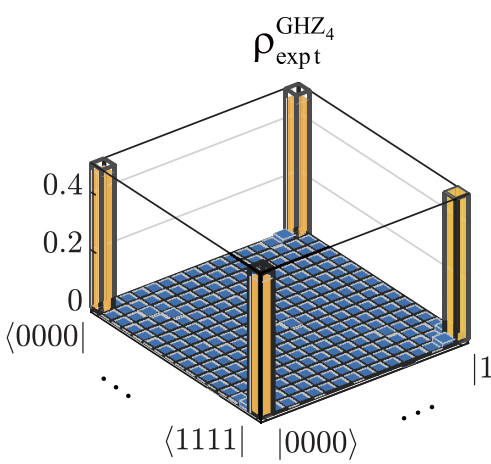

$1111\rangle$

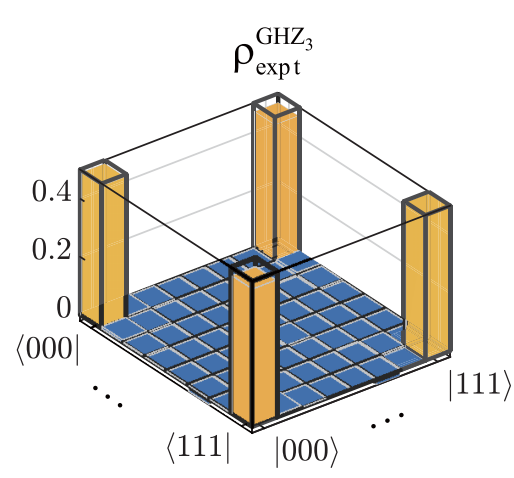

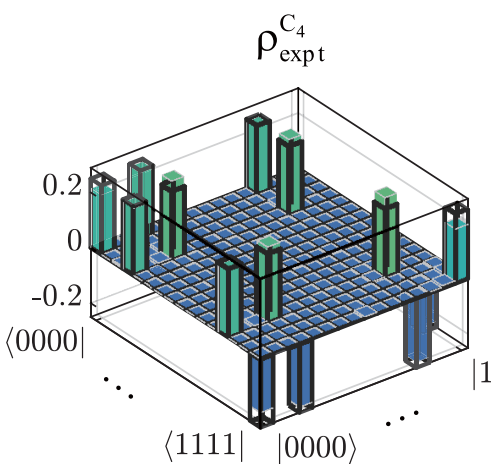

$|1111\rangle$

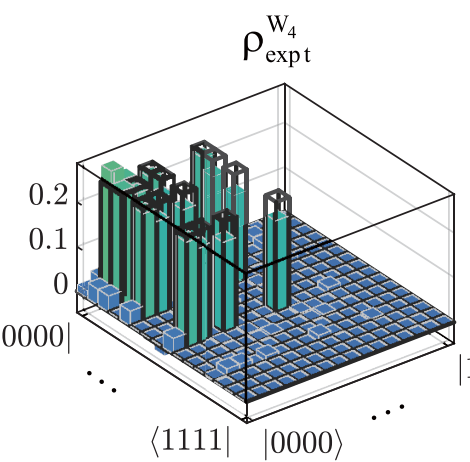

$|1111\rangle$

FIG. 8. Reconstructed density matrices of generated multiqubit states.

$$
\begin{gathered}
\rho_{\mathrm{CB}}^{\mathrm{GHZ}_{4}}=(1-\mu)\left|\mathrm{GHZ}_{4}\right\rangle\left\langle\mathrm{GHZ}_{4}\right|+\frac{\mu}{2}(|0000\rangle\langle 0000|+| 1111\rangle\langle 1111|), \\
\rho_{\mathrm{CB}}^{\mathrm{C}_{4}}=(1-\mu)\left|\mathrm{C}_{4}\right\rangle\left\langle\mathrm{C}_{4}\right|+\frac{\mu}{4}(|0000\rangle\langle 0000|+| 0011\rangle\langle 0011|+| 1100\rangle\langle 1100|+| 1111\rangle\langle 1111|), \\
\rho_{\mathrm{CB}}^{\mathrm{W}_{3}}=(1-\mu)\left|\mathrm{W}_{3}\right\rangle\left\langle\mathrm{W}_{3}\right|+\frac{\mu}{3}(|100\rangle\langle 100|+| 010\rangle\langle 010|+| 001\rangle\langle 001|), \\
\rho_{\mathrm{CB}}^{\mathrm{W}_{4}}=(1-\mu)\left|\mathrm{W}_{4}\right\rangle\left\langle\mathrm{W}_{4}\right|+\frac{\mu}{4}(|1000\rangle\langle 1000|+| 0100\rangle\langle 0100|+| 0010\rangle\langle 0010|+| 0001\rangle\langle 0001|) .
\end{gathered}
$$

In the graph-diagonal basis (GDB),

$$
\begin{gathered}
\rho_{\mathrm{GDB}}^{\mathrm{GHZ}_{3}}=U^{\mathrm{GHZ}_{3}} \rho_{\mathrm{CB}}^{\mathrm{GHZ}_{3}} U^{\mathrm{GHZ}_{3}^{\dagger}}=\sum_{k=1}^{8} \lambda_{k}\left|\psi_{k}^{\mathrm{GHZ}_{3}}\right\rangle\left\langle\psi_{k}^{\mathrm{GHZ}_{3}}\left|=\left(1-\frac{\mu}{2}\right)\right| \psi_{1}^{\mathrm{GHZ}_{3}}\right\rangle\left\langle\psi_{1}^{\mathrm{GHZ}_{3}}\left|+\frac{\mu}{2}\right| \psi_{8}^{\mathrm{GHZ}_{3}}\right\rangle\left\langle\psi_{8}^{\mathrm{GHZ}_{3}}\right|, \\
\rho_{\mathrm{GDB}}^{\mathrm{GHZ}_{4}}=U^{\mathrm{GHZ}_{4}} \rho_{\mathrm{CB}}^{\mathrm{GHZ}_{4}} U^{\mathrm{GHZ}_{4}^{\dagger}}=\sum_{k=1}^{16} \lambda_{k}\left|\psi_{k}^{\mathrm{GHZ}_{4}}\right\rangle\left\langle\psi_{k}^{\mathrm{GHZ}_{4}}\left|=\left(1-\frac{\mu}{2}\right)\right| \psi_{1}^{\mathrm{GHZ}_{4}}\right\rangle\left\langle\psi_{1}^{\mathrm{GHZ}_{4}}\left|+\frac{\mu}{2}\right| \psi_{16}^{\mathrm{GHZ}_{4}}\right\rangle\left\langle\psi_{16}^{\mathrm{GHZ}_{4}}\right|, \\
\rho_{\mathrm{GDB}}^{\mathrm{C}_{4}}=U^{\mathrm{C}_{4}} \rho_{\mathrm{CB}}^{\mathrm{C}_{4}} U^{\mathrm{C}_{4} \dagger}=\sum_{k=1}^{16} \lambda_{k}\left|\psi_{k}^{\mathrm{C}_{4}}\right\rangle\left\langle\psi_{k}^{\mathrm{C}_{4}}\left|=\left(1-\frac{3 \mu}{4}\right)\right| \psi_{1}^{\mathrm{C}_{4}}\right\rangle\left\langle\psi_{1}^{\mathrm{C}_{4}}\right|+\frac{\mu}{4}\left(\left|\psi_{4}^{\mathrm{C}_{4}}\right\rangle\left\langle\psi_{4}^{\mathrm{C}_{4}}|+| \psi_{13}^{\mathrm{C}_{4}}\right\rangle\left\langle\psi_{13}^{\mathrm{C}_{4}}|+| \psi_{16}^{\mathrm{C}_{4}}\right\rangle\left\langle\psi_{16}^{\mathrm{C}_{4}}\right|\right)
\end{gathered}
$$

are still graph-diagonal states under the dephasing channel, which means that the estimated lower bounds on such states are tight, and it is easy to check that $\rho_{\mathrm{GDB}}^{\mathrm{W}_{3}}=U^{\mathrm{W}_{3}} \rho_{\mathrm{CB}}^{\mathrm{W}_{3}} U^{\mathrm{W}_{3}} \neq \sum_{k} \lambda_{k}\left|\psi_{k}^{\mathrm{W}_{3}}\right\rangle\left\langle\psi_{k}^{\mathrm{W}_{3}}\right|$ and $\rho_{\mathrm{GDB}}^{\mathrm{W}_{4}}=U^{\mathrm{W}_{4}} \rho_{\mathrm{CB}}^{\mathrm{W}_{4}} U^{\mathrm{W}_{4} \dagger} \neq \sum_{k} \lambda_{k}\left|\psi_{k}^{\mathrm{W}_{4}}\right\rangle\left\langle\psi_{k}^{\mathrm{W}_{4}}\right|$.

In experiment, we observe that the normalized distance of estimated $l^{c}\left(\rho^{\mathrm{W}_{4}}\right)$ is larger than that of other states as the prepared $\rho_{\text {expt }}^{\mathrm{W}_{4}}$ has lower fidelity compared with other states. More importantly, it is not a graph-diagonal state anymore under the dephasing channel, which causes there to be only a 5\% difference in state fidelity (shown in Appendix B) but a 15\% difference in normalized distance [shown in Fig. 3(c)].

[1] F. Grosshans, G. Van Assche, J. Wenger, R. Brouri, N. J. Cerf, and P. Grangier, Quantum key distribution using gaussianmodulated coherent states, Nature (London) 421, 238 (2003).
[2] P. J. Coles, E. M. Metodiev, and N. Lütkenhaus, Numerical approach for unstructured quantum key distribution, Nat. Commun. 7, 11712 (2016). 
[3] J. Ma, Y. Zhou, X. Yuan, and X. Ma, Operational interpretation of coherence in quantum key distribution, Phys. Rev. A 99, 062325 (2019).

[4] V. Giovannetti, S. Lloyd, and L. Maccone, Advances in quantum metrology, Nat. Photonics 5, 222 (2011).

[5] C. Zhang, T. R. Bromley, Y.-F. Huang, H. Cao, W.-M. Lv, B.-H. Liu, C.-F. Li, G.-C. Guo, M. Cianciaruso, and G. Adesso, Demonstrating Quantum Coherence and Metrology that is Resilient to Transversal Noise, Phys. Rev. Lett. 123, 180504 (2019).

[6] J. Åberg, Catalytic Coherence, Phys. Rev. Lett. 113, 150402 (2014).

[7] M. Lostaglio, D. Jennings, and T. Rudolph, Description of quantum coherence in thermodynamic processes requires constraints beyond free energy, Nat. Commun. 6, 6383 (2015).

[8] V. Narasimhachar and G. Gour, Low-temperature thermodynamics with quantum coherence, Nat. Commun. 6, 7689 (2015).

[9] E. Romero, R. Augulis, V. I. Novoderezhkin, M. Ferretti, J. Thieme, D. Zigmantas, and R. van Grondelle, Quantum coherence in photosynthesis for efficient solar-energy conversion, Nat. Phys. 10, 676 (2014).

[10] V. Vedral, M. B. Plenio, M. A. Rippin, and P. L. Knight, Quantifying Entanglement, Phys. Rev. Lett. 78, 2275 (1997).

[11] V. Vedral and M. B. Plenio, Entanglement measures and purification procedures, Phys. Rev. A 57, 1619 (1998).

[12] E. Chitambar and G. Gour, Quantum resource theories, Rev. Mod. Phys. 91, 025001 (2019).

[13] J. Aberg, Quantifying superposition, arXiv:quant-ph/0612146 [quant-ph].

[14] T. Baumgratz, M. Cramer, and M. B. Plenio, Quantifying Coherence, Phys. Rev. Lett. 113, 140401 (2014).

[15] A. Streltsov, U. Singh, H. S. Dhar, M. N. Bera, and G. Adesso, Measuring Quantum Coherence with Entanglement, Phys. Rev. Lett. 115, 020403 (2015).

[16] C. Napoli, T. R. Bromley, M. Cianciaruso, M. Piani, N. Johnston, and G. Adesso, Robustness of Coherence: An Operational and Observable Measure of Quantum Coherence, Phys. Rev. Lett. 116, 150502 (2016).

[17] K. Bu, U. Singh, S.-M. Fei, A. K. Pati, and J. Wu, Maximum Relative Entropy of Coherence: An Operational Coherence Measure, Phys. Rev. Lett. 119, 150405 (2017).

[18] B. Chen and S.-M. Fei, Notes on modified trace distance measure of coherence, Quantum Inf. Process. 17, 107 (2018).

[19] S. Du, Z. Bai, and X. Qi, Coherence manipulation under incoherent operations, Phys. Rev. A 100, 032313 (2019).

[20] S. Du, Z. Bai, and X. Qi, Coherence measures and optimal conversion for coherent states, Quantum Inf. Comput. 15, 1307 (2015).

[21] C. L. Liu, D.-J. Zhang, X.-D. Yu, Q.-M. Ding, and L. Liu, A new coherence measure based on fidelity, Quantum Inf. Process. 16, 198 (2017)

[22] X. Qi, T. Gao, and F. Yan, Measuring coherence with entanglement concurrence, J. Phys. A: Math. Theor. 50, 285301 (2017).

[23] Z. Xi and S. Yuwen, Coherence measure: Logarithmic coherence number, Phys. Rev. A 99, 022340 (2019).

[24] Z. Xi and S. Yuwen, Epsilon-smooth measure of coherence, Phys. Rev. A 99, 012308 (2019).

[25] Q. Zhao, Y. Liu, X. Yuan, E. Chitambar, and X. Ma, One-Shot Coherence Dilution, Phys. Rev. Lett. 120, 070403 (2018).
[26] Y. Zhou, Q. Zhao, X. Yuan, and X. Ma, Polynomial measure of coherence, New J. Phys. 19, 123033 (2017).

[27] A. Streltsov, E. Chitambar, S. Rana, M. N. Bera, A. Winter, and M. Lewenstein, Entanglement and Coherence in Quantum State Merging, Phys. Rev. Lett. 116, 240405 (2016).

[28] E. Chitambar, A. Streltsov, S. Rana, M. N. Bera, G. Adesso, and M. Lewenstein, Assisted Distillation of Quantum Coherence, Phys. Rev. Lett. 116, 070402 (2016).

[29] A. Streltsov, S. Rana, M. N. Bera, and M. Lewenstein, Towards Resource Theory of Coherence in Distributed Scenarios, Phys. Rev. X 7, 011024 (2017).

[30] E. Chitambar and M.-H. Hsieh, Relating the Resource Theories of Entanglement and Quantum Coherence, Phys. Rev. Lett. 117, 020402 (2016).

[31] D. Girolami and B. Yadin, Witnessing multipartite entanglement by detecting asymmetry, Entropy 19124 (2017).

[32] J. Ma, B. Yadin, D. Girolami, V. Vedral, and M. Gu, Converting Coherence to Quantum Correlations, Phys. Rev. Lett. 116, 160407 (2016).

[33] N. Killoran, F. E. S. Steinhoff, and M. B. Plenio, Converting Nonclassicality into Entanglement, Phys. Rev. Lett. 116, 080402 (2016).

[34] Y.-T. Wang, J.-S. Tang, Z.-Y. Wei, S. Yu, Z.-J. Ke, X.-Y. Xu, C.-F. Li, and G.-C. Guo, Directly Measuring the Degree of Quantum Coherence using Interference Fringes, Phys. Rev. Lett. 118, 020403 (2017).

[35] W. Zheng, Z. Ma, H. Wang, S.-M. Fei, and X. Peng, Experimental Demonstration of Observability and Operability of Robustness of Coherence, Phys. Rev. Lett. 120, 230504 (2018).

[36] G. Smith, J. A. Smolin, X. Yuan, Q. Zhao, D. Girolami, and X. Ma, Quantifying coherence and entanglement via simple measurements, arXiv:1707.09928 [quant-ph].

[37] D.-J. Zhang, C. L. Liu, X.-D. Yu, and D. M. Tong, Estimating Coherence Measures from Limited Experimental Data Available, Phys. Rev. Lett. 120, 170501 (2018).

[38] X.-D. Yu and O. Gühne, Detecting coherence via spectrum estimation, Phys. Rev. A 99, 062310 (2019).

[39] D. Gottesman, Class of quantum error-correcting codes saturating the quantum Hamming bound, Phys. Rev. A 54, 1862 (1996).

[40] D. Gottesman, Stabilizer codes and quantum error correction, Ph.D. thesis, California Institute of Technology, 1997.

[41] A. Winter and D. Yang, Operational Resource Theory of Coherence, Phys. Rev. Lett. 116, 120404 (2016).

[42] Q. Zhao, Y. Liu, X. Yuan, E. Chitambar, and A. Winter, Oneshot coherence distillation: Towards completing the picture, IEEE Trans. Inf. Theory 65, 6441 (2019).

[43] X. Yuan, H. Zhou, Z. Cao, and X. Ma, Intrinsic randomness as a measure of quantum coherence, Phys. Rev. A 92, 022124 (2015).

[44] M. Hayashi and H. Zhu, Secure uniform random-number extraction via incoherent strategies, Phys. Rev. A 97, 012302 (2018).

[45] X. Yuan, Q. Zhao, D. Girolami, and X. Ma, Quantum coherence and intrinsic randomness, Adv. Quantum Technol. 2, 1900053 (2019).

[46] C. A. Rodríguez-Rosario, T. Frauenheim, and A Aspuru-Guzik, Thermodynamics of quantum coherence, arXiv:1308.1245. 
[47] F. Cicalese and U. Vaccaro, Supermodularity and subadditivity properties of the entropy on the majorization lattice, IEEE Trans. Inf. Theory 48, 933 (2002).

[48] M. A. Nielsen and I. L. Chuang, Quantum Computation and Quantum Information, 10th ed. (Cambridge University Press, Cambridge, 2010).

[49] W. Dür, H. Aschauer and H.-J. Briegel, Multiparticle Entanglement Purification for Graph States, Phys. Rev. Lett. 91, 107903 (2003).

[50] O. Gühne, B. Jungnitsch, T. Moroder, and Y. S. Weinstein, Multiparticle entanglement in graph-diagonal states: Necessary and sufficient conditions for four qubits, Phys. Rev. A 84, 052319 (2011).

[51] D. Schlingemann, Stabilizer codes can be realized as graph codes, arXiv:quant-ph/0111080.

[52] Y. Yao, X. Xiao, L. Ge, and C. P. Sun, Quantum coherence in multipartite systems, Phys. Rev. A 92, 022112 (2015).

[53] J. I. de Vicente and A. Streltsov, Genuine quantum coherence, J. Phys. A: Math. Theor. 50, 045301 (2017).

[54] C. L. Liu, Y.-Q. Guo, and D. M. Tong, Enhancing coherence of a state by stochastic strictly incoherent operations, Phys. Rev. A 96, 062325 (2017).

[55] C. L. Liu and D. L. Zhou, Deterministic Coherence Distillation, Phys. Rev. Lett. 123, 070402 (2019).

[56] T. Kim, M. Fiorentino, and F. N. C. Wong, Phase-stable source of polarization-entangled photons using a polarization Sagnac interferometer, Phys. Rev. A 73, 012316 (2006).

[57] K. Chen, C.-M. Li, Q. Zhang, Y.-A. Chen, A. Goebel, S. Chen, A. Mair, and J.-W. Pan, Experimental Realization of OneWay Quantum Computing with Two-Photon Four-Qubit Cluster States, Phys. Rev. Lett. 99, 120503 (2007).
[58] G. Vallone, E. Pomarico, F. De Martini, and P. Mataloni, Active One-Way Quantum Computation with Two-Photon Four-Qubit Cluster States, Phys. Rev. Lett. 100, 160502 (2008).

[59] W.-B. Gao, C.-Y. Lu, X.-C. Yao, P. Xu, O. Gühne, A. Goebel, Y.-A. Chen, C.-Z. Peng, Z.-B. Chen, and J.-W. Pan, Experimental demonstration of a hyper-entangled ten-qubit Schrödinger cat state, Nat. Phys. 6, 331 (2010).

[60] G. Tóth and O. Gühne, Entanglement detection in the stabilizer formalism, Phys. Rev. A 72, 022340 (2005).

[61] Q. Zhao and Y. Zhou, Constructing multipartite Bell inequalities from stabilizers, arXiv:2002.01843 [quant-ph].

[62] D. Gottesman, Fault-tolerant quantum computation with higherdimensional systems, in Quantum Computing and Quantum Communications, edited by C. P. Williams (Springer, Berlin, 1999), pp. 302-313.

[63] E. Hostens, J. Dehaene, and B. De Moor, Stabilizer states and Clifford operations for systems of arbitrary dimensions and modular arithmetic, Phys. Rev. A 71, 042315 (2005).

[64] V. Gheorghiu, Standard form of qudit stabilizer groups, Phys. Lett. A 378, 505 (2014).

[65] A. K. Pati, U. Singh, and U. Sinha, Measuring nonHermitian operators via weak values, Phys. Rev. A 92, 052120 (2015).

[66] G. Nirala, S. N. Sahoo, A. K. Pati, and U. Sinha, Measuring average of non-Hermitian operator with weak value in a MachZehnder interferometer, Phys. Rev. A 99, 022111 (2019).

[67] Y. Dai, Y. Dong, Z. Xu, W. You, C. Zhang, and O. Gühne, Experimentally Accessible Lower Bounds for Genuine Multipartite Entanglement and Coherence Measures, Phys. Rev. Appl. 13, 054022 (2020). 